\title{
OPTIMIZING THE CONTAINER TRUCK PATHS WITH UNCERTAIN TRAVEL TIME IN CONTAINER PORTS
}

\author{
Jiaming LIU ${ }^{1}$, Bin YU ${ }^{1,2}$, Wenxuan $\mathrm{SHAN}^{1}$, Baozhen $\mathrm{YAO}^{3 *}$, Yao SUN ${ }^{4}$ \\ ${ }^{1}$ School of Transportation Science and Engineering, Beihang University, Beijing, China \\ ${ }^{2}$ Advanced Innovation Center for Big Data and Brain Computing, Beihang University, Beijing, China \\ ${ }^{3}$ State Key Laboratory of Structural Analysis for Industrial Equipment, School of Automotive Engineering, \\ Dalian University of Technology, Dalian, China \\ ${ }^{4}$ Tianjin Municipal Engineering Design and Research Institute, Tianjin, China
}

Submitted 26 September 2019; resubmitted 25 November 2019, 7 April 2020; accepted 5 July 2020

\begin{abstract}
The yard template problem in container ports determines the assignment of space to store containers for the vessels, which could impact container truck paths. Actually, the travel time of container truck paths is uncertain. This paper considers the uncertainty from two perspectives: (1) the yard congestion in the context of yard truck interruptions, (2) the correlation among adjacent road sections (links). A mixed-integer programming model is proposed to minimize the travel time of container trucks. The reliable shortest path, which takes the correlation among links into account is firstly discussed. To settle the problem, a Shuffled Complex Evolution Approach (SCE-UA) algorithm is designed to work out the assignment of yard template, and the $\mathrm{A}^{*}$ algorithm is presented to find the reliable shortest path according to the port operator's attitude. In our case study, one yard in Dalian (China) container port is chosen to test the applicability of the model. The result shows the proposed model can save $9 \%$ of the travel time of container trucks, compared with the model without considering the correlation among adjacent links.
\end{abstract}

Keywords: container port, yard template, reliable shortest path, SCE-UA algorithm.

\section{Abbreviations}

AGV - automatic guided vehicle;

AVG - average result;

$\mathrm{BR}$ - best result;

CCE - competitive complex evolution;

$\mathrm{CDF}$ - cumulative distribution function;

CPLEX - IBM ILOG CPLEX optimizer;

CPU - central processing unit;

GDP - gross domestic product;

NON-CORR - non-correlation;

OCTP-UTT - optimizing container truck paths with uncertain travel time;

QC - quay crane;

RGS - route guidance system;

RSPP - reliable shortest path problem;

SCE-UA - shuffled complex evolution approach;

SD - standard deviation;

SWO - squeaky wheel optimization;

TEU - twenty-foot equivalent unit.

\section{Introduction}

\section{Background}

Compared with the growth rate of world GDP, the amount of container transportation has increased about three times (Meng et al. 2014). As lots of containers are stored and transhipped among ports every day, the turnaround time of containers is influenced by the operation efficiency of ports. It is essential to improve the operation efficiency and maximize the throughput because the profit of a port is relevant to the number of handled containers (Chang et al. 2010). There are many factors impacting the operation efficiency, such as the berth and yard operation (Jin et al. 2015). In the past decades, the operation efficiency of ports in the berth side has been improved significantly because of the advanced technologies (e.g., indented berths and the double forty-foot QCs) and management (berth allocation, QC assignment and scheduling) - Lee and Jin (2013), Zhen (2015). While the yard side may become a bottleneck that hinders the operation efficiency, especially

*Corresponding author. E-mail: yaobaozhen@dlut.edu.cn 
in the ports that have a large number of QCs. Therefore, the management of the yard side is crucial to promote the port's competitiveness on global shipping market.

The yard template planning is a concept of planning the yard in container ports (Moorthy, Teo 2006), which is concerned with the assignment of yard storage locations (subblocks). It aims to minimize the total cost of moving containers from berth or gate to subblocks and vice versa. One way is to minimize the total distance of moving containers, but it could hardly improve the situation in practice. Although the travel distance is minimized, the container trucks may waste lots of time when there has congestion along the path. Actually, the yard congestion is common in reality. Nowadays, multi-level stacking is universal in yards with heavy traffic. It may lead to high concentration of activities within a small area and cause yard traffic congestion (Han et al. 2008). When there are too many container trucks running along a link or passing through a cross at the same time, the cruising speed of these trucks will be affected by each other. In this condition, the trucks have to slow down or even stop when they come across yard congestion. Moreover, the congestion on a link could easily transfer to adjacent links according to the traffic flow theory. That is, there exists correlation among adjacent links. The travel time of a container truck is uncertain with the consideration of yard congestion on some link and the impact of adjacent links. This is the reason why the optimized distance is not equivalent to the optimized travel time. In this paper, our motivation is to minimize the travel time and find a reliable shortest path for container trucks considering the uncertain travel time.

\section{Literature review}

There are abundant studies on container allocation, berth allocation and crane assignment (Zhang et al. 2003; Fan et al. 2012; Maloni, Paul 2013; Peng et al. 2016). Kim and Bae (1998) discussed to reallocate export containers to the best organization for loading vessels. They used a hierarchical approach to divide the problem into three sub-problems, bay matching, move planning and task sequencing. Zhang et al. (2003) studied how to allocate storage space in the yards considering the mixture of import and export containers. They divided the allocation problem into two steps: (1) all of the containers were placed in a determined storage block, (2) all the containers were allocated to minimize the total travel distance. Kim, K. H. and Kim, K. Y. (2007) presented a method to determine the minimum price for storing the containers in a yard. In addition, the storage charge urged the customers to store their containers only for a short time so as to relieve congestion. Zhen et al. (2011) proposed an integrated model, which considered berth allocation and yard template planning simultaneously, these two problems fit well with each other. Following the study in 2011, Zhen (2015) formulated a robust problem of berth allocation under uncertain environment. The factor of periodicity had been explicitly considered in the stochastic programming formulation model and the robust formulation model. Jin et al. (2015) proposed the yard crane profile that was used in an optimization model on storage deployment and management. Zhen et al. (2019) studied an integrated optimization problem on QC and yard truck scheduling in container terminals, which showed good results.

Besides, the transhipment tends to be increasingly important, both in contemporary and forthcoming future. Many researchers have studied transhipment management in container ports. Lee and Jin (2013) settled three tactical decision problems simultaneously for a container transhipment terminal considering the quayside congestion and the cost of container movements. Moccia et al. (2009) came up with a method based on column generation for allocating containers in transhipment ports. Nishimura et al. (2009) developed an optimization model that aimed to minimize the time of moving containers and dwell time. Zhen (2013) proposed a mixed-integer programming model to minimize the expected route length of containers flows considering the uncertain berthing time and position. A heuristic algorithm was developed to solve the large-scale instance. Wang et al. (2015) proposed the container assignment model based on profit maximization, considering transhipment under liner shipping networks, a segmentation procedure was developed to accelerate the algorithm.

The container allocation in the yard affects the travel time of the container truck as different assignments result in different container truck paths. Some researchers have investigated the container truck routing and scheduling problem (Vis, De Koster 2003; Kaveshgar, Huynh 2015; He et al. 2015; Chen et al. 2019; Shan et al. 2019). Vis and De Koster (2003) reviewed early works of container transportation from ship to yard and vice versa. Each truck was assigned to a path to complete the transportation task. Nishimura et al. (2005) focused on the trailer routing problem. The dynamic routing was proposed to reduce the travel distance. Cao et al. (2010) proposed an integrated model for yard truck and yard crane operation. The Bender's decomposition was used to solve the model. Chen et al. (2011) studied the truck transportation in the container terminal. The multiple truck routing problem was solved based on the transportation tasks. Chen et al. (2013) proposed a nonlinear programming model to analyse time-dependent truck queuing process in which stochastic service time distributions at gates and yards of a container terminal were considered. $\mathrm{Lu}$ and Le (2014) studied the integration of yard carne, QC and yard truck scheduling problem with uncertain factors. They assumed that the yard truck driving time was subject to normal distribution according to the statistics of Shanghai port, which showed good results. Kaveshgar and Huynh (2015) considered the yard truck scheduling problem from the real-world operational instances such as precedence degree of containers and QC safety margin. A mixed-integer programming model was formulated to work out the problem. He et al. (2015) integrated three factors that would impact the yard efficiency, QC, yard truck and yard crane. Shan et al. (2019) considered a facility location and truck routing problem from the supply chain perspective. 
A heuristic algorithm was used to solve the problem because the model was complicated.

In recent years, scholars try to develop effective shortest path algorithms for RGS (Huang et al. 2007; Zeng, Church 2009; Yu et al. 2018; Peng et al. 2020). Most RGSs assume the travel time on the link is deterministic. However, link travel time seems to be highly stochastic in yard networks due to the complex port operation and spatial correlation (Chan et al. 2009; Yao et al. 2019a, 2019b). For instance, a yard congestion happening on a link may cause travel delays on adjacent links as well. Besides, yard cranes move from side to side on a link may also cause the travel delays on other links. Therefore, researchers have developed methods to solve the RSPP. Shao et al. (2004) presented a metaheuristic algorithm to find the reliable shortest path considering the travel time and variance. Chen and Ji (2005) proposed the alpha-shortest path that aimed to minimize the total travel time in a certain confidence level. Nikolova (2009) developed a quantification method to find the reliable shortest path for passengers with different objectives. Nie and $\mathrm{Wu}$ (2009) proposed a novel model, which could generate nondominated paths in which the path could not be replaced by others.

Previous works have made great efforts to improve the yard operation efficiency. Zhen (2016) first discussed the notion of yard congestion in the context of yard truck interruptions and developed a combination of probabilistic and physics-based models for truck interruptions. Inspired by his work on truck interruptions, we propose a model to optimize the reliable container truck paths with uncertain travel time.

\section{Contribution}

The contribution of this paper are as follows:

"॥ first, this paper develops a model to optimize the path travel time for container trucks and find a reliable shortest path by considering the yard congestion and the impact of adjacent links. The variance and covariance among links are introduced to describe the uncertainty of travel time. From our case study, the yard congestion and impact among adjacent links truly affect the actual travel time of container trucks in ports;

"» second, this paper considers the probability of arriving at the destination within the expected travel time. In different situations, the attitude of port operators toward the risk of being late is changeable. In this paper, the on-time arrival probability $\alpha$ which represents port operators' attitude toward risk of being late is presented. Port operators characterized by risk-averse, risk-neutral, and risk-seeking could choose the routing strategy freely.

The remainder of this paper is organized as follows:

"» section 1 describes the problem;

"») section 2 is divided into 2 subsections: subsection

2.1 describes the basic work for mathematical formulation and subsection 2.2 formulates the model;
"» a solution method is developed in section 3;

"m) results of the cases are shown in section 4;

"» some conclusions are summarized in the last section.

\section{Problem description}

In the yard side, the container truck path is influenced by the yard template planning, which assigns the container flows between vessels and subblocks. Without considering the container truck path, the yard template planning is a general assignment problem that can be well settled. However, because a large number of container trucks running along the path may cause traffic congestion, the travel time of each truck path is uncertain in reality, especially in the cross that two paths intersect or in the link that two container trucks merge. Here, a typical circumstance in a transhipment port is taken as an example.

In Figure 1, Vessel 1 performs the loading and unloading process. The unloading path is illustrated by the dashed line. The containers to be loaded on other vessels (i.e., Vessel 2, Vessel 3) in future are sent to the subblocks, i.e., S51, S69, S121 for vessel 2 and S21, S74, S109, S114 for Vessel 3. The solid lines refer to the loading paths. The containers from Vessel 2 reserved in the subblocks (i.e., S82, S115, S138) are loaded on Vessel 1. In practice, the travel time of each path is influenced by traffic flow in the yard. In addition, traffic congestion happens commonly, especially at the cross or around the yard crane. Moreover, the link with traffic congestion may also affect the travel time of the adjacent links. In this paper, we consider the influence of traffic flow by: (1) a congestion model in the context of truck interruptions that formulate the travel time of a single link, and (2) the correlation among adjacent links that formulate the travel time of a whole path.

Note that the working range of a QC is limited. When the coming vessel is large, there are probably more than one QC serving the vessel. Thus, the loading/unloading path from different QCs may be different. For simplicity, we assume the berth position of the vessel, which is used to determine the loading/unloading path, is the vessel's middle point.

\section{Optimizing container truck paths with uncertain travel time}

\subsection{Basic work for model formulation}

To formulate the mathematical model, some basic work including formulating the travel time of a single link in the congestion model (subsection 2.1.2), considering the correlation among adjacent links (subsection 2.1.3), finding the reliable shortest paths (subsection 2.1.4), balancing the workload protocol (subsection 2.1.5) should be done in advance.

\subsubsection{The yard network}

In Figure 2, the yard network could be denoted by a directed graph with nodes and links, which is referred to Zhen (2016). Let $G=(N, A, \Psi)$ be a directed graph con- 
sisting of a set of nodes $N$, a set of links $A$, and a set of movements $\Psi$. Figure 2 has three kinds of node, which are berth node, cross node, and subblock node. Berth node and subblock node respectively represents the berth position and subblock, which is denoted by $o \in N$ or $d \in N$ according to the direction of container flow. Cross node represents the crossing in practice. Container trucks at cross node could turn or pass through. A link $a=(i, j) \in A$ has a predecessor node $i \in N$ and a successor node $j \in N$. The container truck path is made up of a series of consecutive links from node $o$ to node $d$. In this paper, index $o$ and $d$ specially refer to the origin and destination of a path, while $(i, j)$ refers to a link in the path. $\psi_{i, j, k}=((i, j),(j, k)) \in \Psi$ denotes an allowed movement (e.g., turn or pass through movement) at node $j . \psi_{i, j, k} \in \Psi$ means that the movement has to be carried out at the middle node $j$. Here we take the unloading process as an instruction. In Figure 2, the blue line represents one of the paths from the berth $o$ to the subblock $d$, let $\Omega_{u}^{o, d}=\left\{a_{1}, \ldots, a_{m}, \ldots, a_{\lambda}\right\}$ be the $u$ path from $o$ to $d$, consisting of $\lambda$ consecutive links. We define the path travel time as $t_{u}^{o, d}$, which is a sum of link travel time, as is shown in Equation (1). Considering the uncertainty of path travel time, $t_{u}^{o, d}$ is a random variable that we will talk detail in section 2.1.3:

$$
t_{u}^{o, d}=\sum_{m=1}^{\lambda} t_{a_{m}},
$$

where: $t_{a_{m}}$ is the travel time of $a_{m}$ (the $m$ th link of path $u$ ).

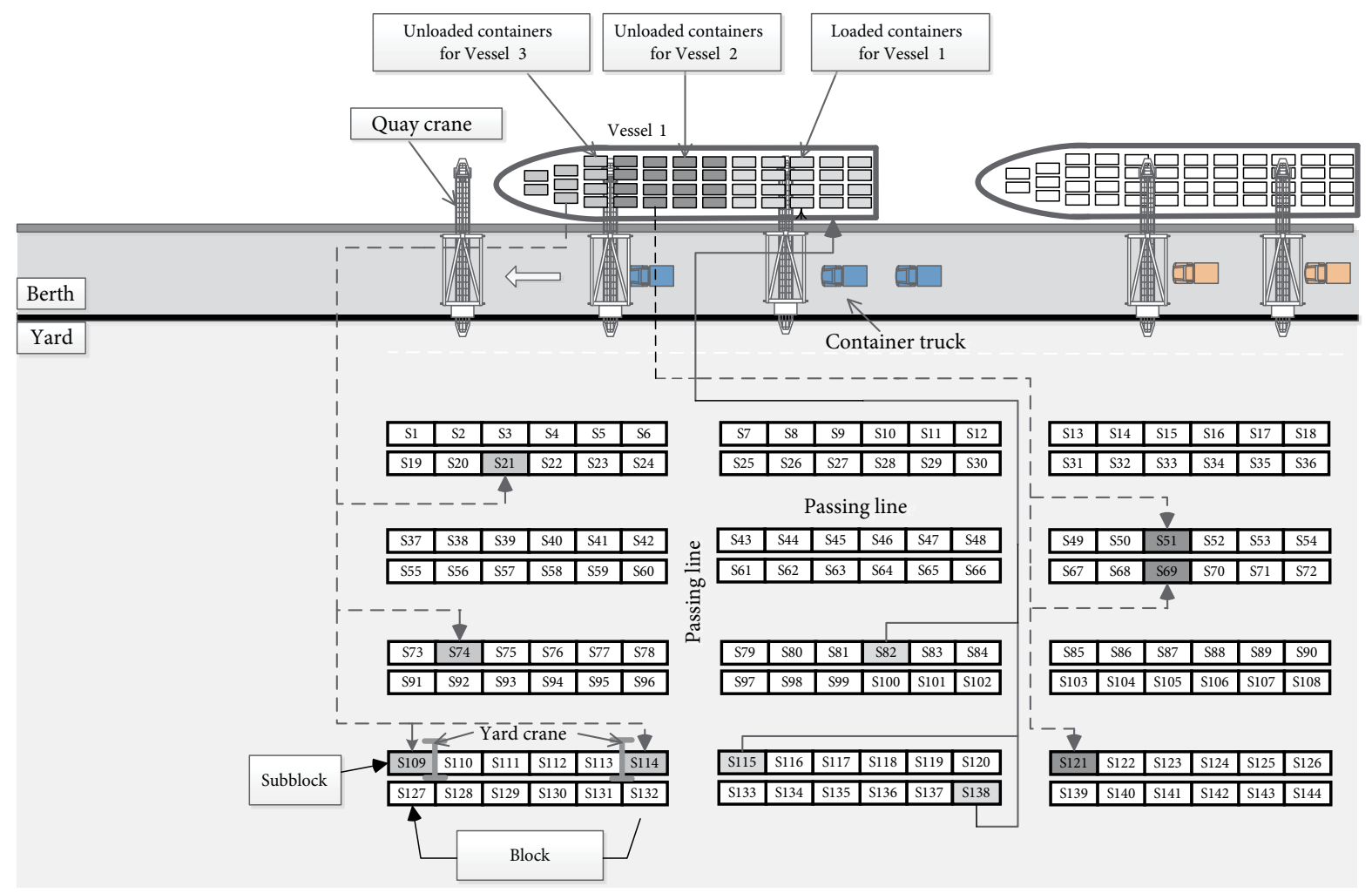

Figure 1. A typical working process of container ports

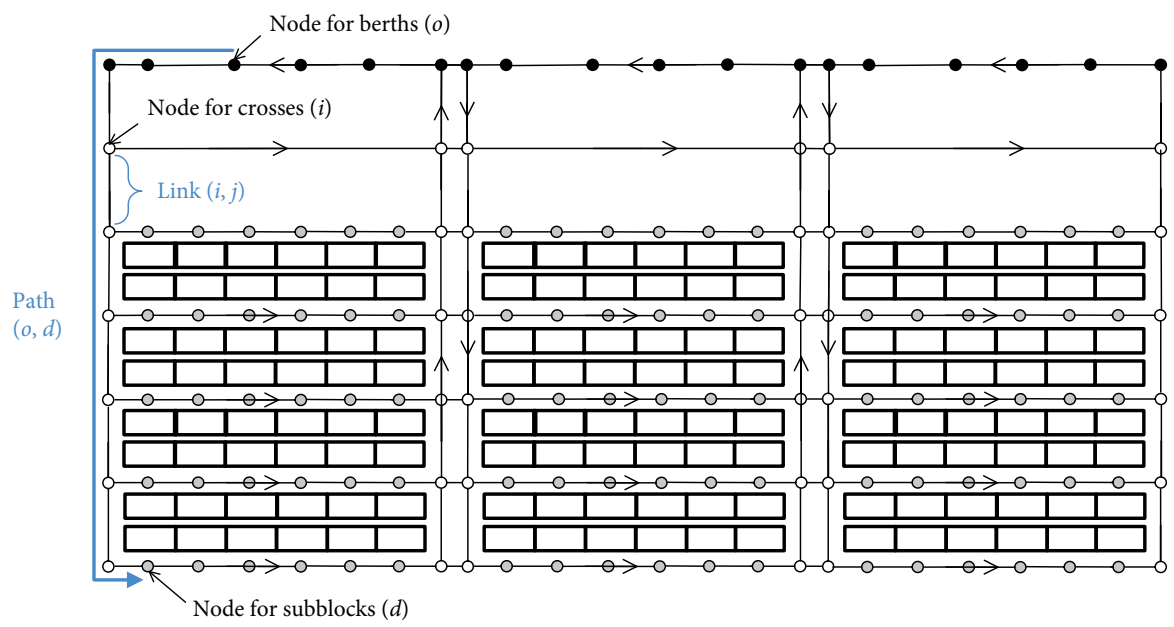

Figure 2. The yard network denoted by nodes and links 
There are probably more than one path between the origin and destination, but it should be noted that paths in the yard network should follow the traffic rule. In this paper, the truck is guided by an anticlockwise direction, as shown in Figure 2. There are two truck lanes between every two adjacent blocks.

\subsubsection{Congestion model of link travel time}

As above mentioned, the link travel time is uncertain considering the yard congestion. The yard congestion prevents container trucks from traveling freely or prevents AGVs from running freely in the ports (Roy et al. 2016). If excessive container trucks are passing through the crossroad or running along a narrow lane at the same time, the normal speed of container trucks will be affected by each other. On this condition, the container trucks are forced to slow down or even stop when they are interrupted during the transportation (Zhang et al. 2009).

Figure 3 shows two common examples of the truck interruption happened in the yard, which is referred to Zhen (2016). As shown in Figure 3a, when a container truck (Truck 1) is running along the link, at the same time some other container trucks are running from the inside lane to the main lane, Truck 1 may slow down or stop to avoid collisions. The other type of interruption is shown in Figure $3 \mathrm{~b}$. When Truck 1 is running along the link, at the same time some other container trucks are running from passing line 1, 2 or 3 to passing line 4, Truck 1 may slow down or stop to avoid collisions.

Here we employ Zhen (2016) to formulate the influence of traffic flow to the link travel time. The travel time is affected by the number of truck interruptions on a link. Let $t_{i, j}(r)$ be the expected travel time of the link $(i, j)$ given $r$ interruptions, and let $P(r)_{i, j}$ be the probability of occurring $r$ interruptions on link $(i, j)$. The expected travel time of passing through link $(i, j)$ can be calculated as:

$$
t_{i, j}=\sum_{r=0}^{+\infty} P(r)_{i, j} \cdot t_{i, j}(r)
$$

Referring to the interruption model on a link (Zhang et al. 2009), the probability $P(r)_{i, j}$ is formulated as:

$$
P(r)_{i, j}=\frac{(\bar{R})^{r} \cdot e^{-\bar{R}}}{r !},
$$

where: $\bar{R}$ is a mean number of the interruptions that would influence the transportation of container trucks.

Parameter $\bar{R}$ is calculated by the formula:

$$
\bar{R}=\frac{s_{i, j} \cdot t_{i, j} \cdot v^{2}}{4 \cdot a \cdot d_{i, j} \cdot e_{i, j} \cdot h_{Y C}},
$$

where: $s_{i, j}$ denotes the number of the working subblocks on link $(i, j) ; v$ denotes the average speed of container trucks; $a$ denotes the acceleration (deceleration) of container trucks; $d_{i, j}$ denotes the length of the link $(i, j) ; e_{i, j}$ denotes the number of lanes on link $(i, j) ; h_{Y C}$ denotes the average handling time of a yard crane for a container.

Then, $t_{i, j}(r)$ can be calculated by:

$$
t_{i, j}(r)=\frac{d_{i, j}}{v}+\frac{v_{i, j}^{\prime 2}+v_{i, j}^{\prime \prime 2}}{2 a \cdot v}+\frac{v-v_{i, j}^{\prime}-v_{i, j}^{\prime}}{a}+\frac{r \cdot v}{8 \cdot a},
$$

where: $v_{i, j}^{\prime}, v_{i, j}^{\prime \prime}$ are the speed of trucks at the beginning and end of link $(i, j)$.

The expected travel time of link $(i, j)$ is calculated by:

$$
t_{i, j}=\sum_{r=0}^{+\infty} P(r)_{i, j} \cdot t_{i, j}(r)=\frac{8 \cdot \Gamma}{8 \cdot a-s_{i, j} \cdot \Delta}
$$

where:

$$
\begin{aligned}
\Gamma & =\frac{2 \cdot a \cdot d_{i, j}+v_{i, j}^{\prime 2}+v_{i, j}^{\prime \prime 2}}{2 \cdot v}+v-v_{i, j}^{\prime}-v_{i, j}^{\prime \prime} ; \\
\Delta & =\frac{v^{3}}{4 \cdot a \cdot d_{i . j} \cdot e_{i, j} \cdot h_{Y C}} .
\end{aligned}
$$

Equation (5) indicates that the link travel time is related to $s_{i, j}$ considering the yard congestion. While $s_{i, j}$ is a decision variable, which can be determined by the yard template planning, the travel time of link $(i, j)$ in the yard template planning is correlated with $s_{i, j}$. As $t_{i, j}$ depends on $s_{i, j}$, it can be denoted as $t_{i, j, s}$. Here, $t_{i, j, s}$ means the travel time of link $(i, j)$ when there are $s$ working subblocks on link $(i, j)$. The formulation of link travel time considering yard congestion is validated by a large number of simulation a)

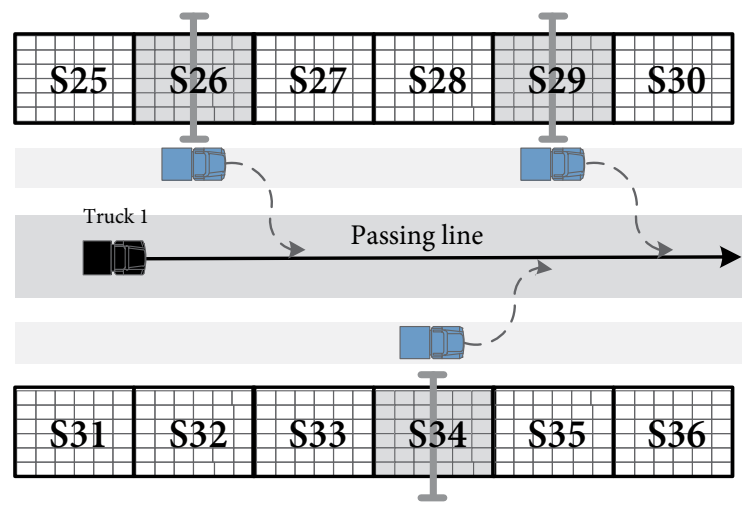

b)

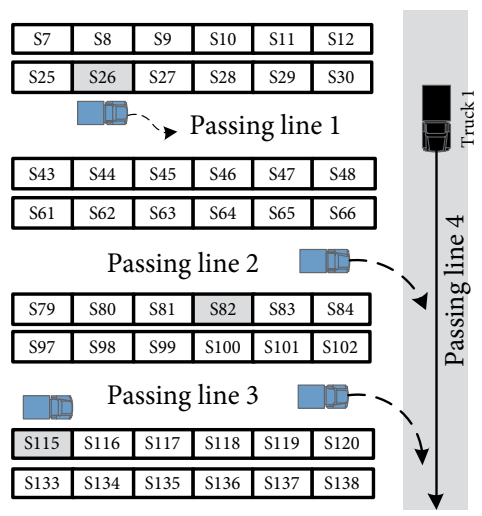

Figure 3. Interruptions happened in the travel path 
runs, all the average relative deviation between simulated results and theoretical results calculated by Equation (5) is lower than 1.5\%. For more proof information about Equations (2)-(5), we suggest the readers refer to Zhen (2016).

\subsubsection{Congestion model considering the correlation among adjacent links}

In fact, the link that is congested may have an influence on the adjacent links. We take Figure $3 \mathrm{~b}$ for an example, any congestion happened in passing line (1 3) may induce the congestion in passing line 4 as well. In addition, the drivers may change their path to the destination so as to save the travel time. To formulate the correlation among adjacent links, we use $k$-neighbouring links of link $(i, j)$, which means the travel time of link $(i, j)$ is related to $k$ neighbouring links from a spatial perspective. Let $X_{i, j}^{q, w}$ be the topological distance (a value that reflects the closeness of the two links) between link $(i, j)$ and link $(g, w)$, e.g. the topological distance of two directly connected links is 1. A link $(g, w)$ is said to be a $k$-neighbouring link of link $(i, j)$ if and only if $X_{i, j}^{q, w}=k . k$ can be valued through a sensitive analysis.

In this paper, we assume that the link travel time follows normal distribution, which is common to see in the studies of RSPP (Chang et al. 2005; Chen et al. 2012, 2014). The expectation of link travel time $t_{i, j, s}$ can be calculated by the congestion model in subsection 2.1.2. And the SD of the link $\sigma_{m}$ can be calculated by statistical data of the yard. In practice, $\sigma_{m}$ means the dispersion of the $m$ th link travel time. The larger $\sigma_{m}$ is, the greater it impacts the adjacent links. Therefore, the expectation of the path travel time $t_{u}^{o, d}$ and the SD of the path travel time $\sigma_{u}^{o, d}$ can be calculated as:

$$
\begin{aligned}
& t_{u}^{o, d}=\sum_{(i, j) \in u} t_{i, j, s} ; \\
& \sigma_{u}^{o, d}=\sqrt{\sum_{m=1}^{\lambda} \sigma_{m}^{2}+\sum_{n=1}^{k} \sum_{m=1}^{\lambda-n} 2 \cdot \operatorname{cov}\left(a_{m}, a_{m+n}\right)},
\end{aligned}
$$

where: $\operatorname{cov}\left(a_{m}, a_{m+n}\right)$ is the covariance of travel time between link $a_{m}$ and $a_{m+n} ; k$ is the closeness degree.

Let $F_{u}^{o, d}(\alpha)$ be the CDF of path travel time $t_{u}^{o, d}$ at $\alpha$ confidence level. So the path travel time can be expressed by the inverse of $\mathrm{CDF}$ :

$$
F_{u}^{o, d}(\alpha)^{-1}=t_{u}^{o, d}+z_{\alpha} \cdot \sigma_{u}^{o, d},
$$

where: $z_{\alpha}$ is the value of standard normal distribution at $\alpha$ confidence level.

Equations (6)-(8) are referred to Chen et al. (2012), which proves the effectiveness of the formulas. Here, the confidence level $\alpha \in(0,1)$ is the probability that the container truck arrives at the destination within the travel time $F_{u}^{o, d}(\alpha)^{-1}$. The on-time arrival probability represents port operators' attitudes toward the risk of being late $(\alpha<0.5, \alpha=0.5$ and $\alpha>0.5$ indicate risk-seeking, riskneutral, and risk-averse attitudes, respectively). The value of $\alpha$ can be determined based on port operator's purpose.

\subsubsection{Find the reliable shortest path}

Now, given the origin $o$, destination $d$, and on-time arrival probability $\alpha$, we can find the reliable shortest path according to Chen and Ji (2005).

$$
\min \sum_{o \in N} \sum_{d \in N} F_{u}^{o, d}(\alpha)^{-1}
$$

subject to Equation (8), and

$$
\begin{aligned}
& t_{u}^{o, d}=\sum_{(i, j) \in A} t_{i, j, s} \cdot x_{i, j}^{o, d} ; \\
& \sum_{j \in N} x_{i, j}^{o, d}-\sum_{k \in N} x_{k, i}^{o, d}= \begin{cases}1, & \forall i=o ; \\
0, & \forall i \neq o, i \neq d ; \\
-1 & \forall i=d ;\end{cases} \\
& x_{i, j}^{o, d} \in\{0,1\}, \forall a_{i j} \in A ; \\
& \psi_{i, j, k} \in \Psi, \forall(i, j) \in \Omega_{u}^{o, d}, \forall(j, k) \in \Omega_{u}^{o, d},
\end{aligned}
$$

where: the decision variable $x_{i, j}^{o, d}$ is regarded as the relationship between link-path; $x_{i, j}^{o, d}=1$ denotes that $a_{i j}$ link $(i, j)$ is in the path $\Omega_{u}^{o d}$, otherwise $x_{i, j}^{o, d}=0$. Equation $(9)$ is to minimize the travel time of all feasible paths. Equations (8) and (10) define the path travel time. Equation (11) guarantees the feasibility of the path. Constraint (12) should be a binary variable concerned with the link-path. Constraint (13) ensures the feasibility of all the movements in the reliable shortest path.

Here a small example is given to show the influence of correlation links to the shortest path. In Figure 4, the travel time of the links obeys normal distribution. The number on the link denotes the average link travel time. The variance and covariance of the link travel time are shown in the matrix. In the variance-covariance matrix, elements on the diagonal line are the variance of the link travel time and off-diagonal elements are the covariance of the travel time between two links. Considering the matrix is symmetric, only a triangular matrix is shown.

In Figure 4, there are three paths $\Omega_{1}^{15}=a_{14} \cup a_{45}$, $\Omega_{2}^{15}=a_{13} \cup a_{35}$, and $\Omega_{3}^{15}=a_{12} \cup a_{23} \cup a_{35}$ from the Node 1 to the Node 5. $\bigcup$ is a path connector $\left(\Omega_{1}^{15}=a_{14} \cup a_{45}\right.$ indicates $\Omega_{1}^{15}$ passes $a_{14}$ and $\left.a_{45}\right)$. From Table 1 , when $\alpha=$ $0.1\left(Z_{\alpha}=-1.28\right)$, the port operators would like to choose path $\Omega_{3}^{15}$, in which the travel time variation is large, to get a small travel time $F_{3}^{15}(\alpha)^{-1}=5.02$. When $\alpha=0.5$ $\left(Z_{\alpha}=0\right)$, the port operators tend to choose path $\Omega_{2}^{15}$, which has the smallest mean travel time $t_{2}^{15}=10$. When $\alpha=0.9\left(Z_{\alpha}=1.28\right)$, the port operators are risk-averse. They prefer to use the more reliable path $\Omega_{1}^{15}$ with a small travel time SD and a larger travel time $F_{1}^{15}(\alpha)^{-1}=14.22$. In results, the optimal solution of the reliable shortest path depends on the attitudes of port operators.

Table 1 . The results of illustrative example

\begin{tabular}{|c|c|c|c|}
\hline$\alpha$ & $F_{1}^{15}(\alpha)^{-1}$ & $F_{2}^{15}(\alpha)^{-1}$ & $F_{3}^{15}(\alpha)^{-1}$ \\
\hline 0.1 & 9.78 & 5.46 & $\mathbf{5 . 0 2}$ \\
\hline 0.5 & 12 & $\mathbf{1 0}$ & 11 \\
\hline 0.9 & 14.22 & 14.54 & 16.98 \\
\hline
\end{tabular}




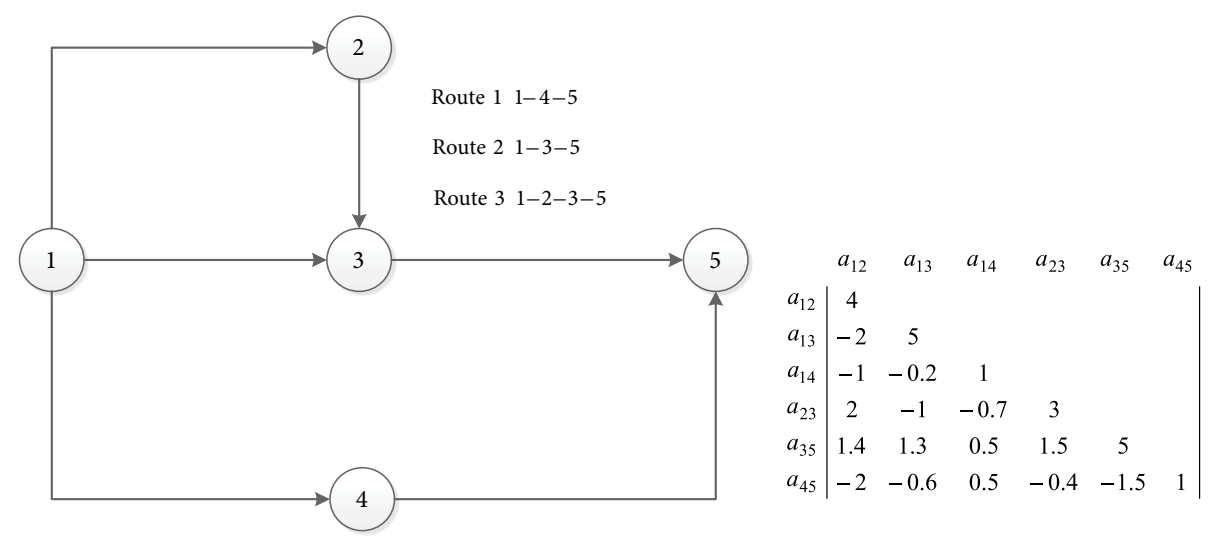

Figure 4. An illustrative example:

$$
\begin{aligned}
& F_{1}^{15}(\alpha)^{-1}=F^{a_{14} \cup a_{45}}(\alpha)^{-1}=5+7+Z_{\alpha} \cdot \sqrt{1+1+2 \cdot 0.5} ; \\
& F_{2}^{15}(\alpha)^{-1}=F^{a_{13} \cup a_{35}}(\alpha)^{-1}=4+6+Z_{\alpha} \cdot \sqrt{5+5+2 \cdot 1.3} \text {; } \\
& F_{3}^{15}(\alpha)^{-1}=F^{a_{12}} \cup a_{23} \cup a_{35}(\alpha)^{-1}=2+3+6+Z_{\alpha} \cdot \sqrt{4+3+5+2 \cdot 2+2 \cdot 1.5+2 \cdot 1.4}
\end{aligned}
$$

\subsubsection{Workload balancing protocol}

In container yard, if two neighbouring subblocks simultaneously have loading or unloading activities, they may accumulate a large number of container trucks in the same lane, which could easily cause traffic congestion. To mitigate the congestion in the planning period, we employ a commonly used high-low workload protocol (Lee et al. 2006; Han et al. 2008; Jiang et al. 2012). Workload means the number of containers handled by a yard crane in a time unit. The high workload is defined as a range, e.g. $[10,20)$, which could not contain the range of low workload, e.g. $[0,10)$. The idea is that two neighbouring subblocks should not simultaneously be in high workload. The judgement of neighbourhood between two subblocks is done by a vicinity matrix used in Lee et al. (2006). Here, a subblock is the neighbour of another one only if they are adjacent and share the same lane. For example, S56 and S57 are neighbours, but S56 and S38 are not neighbours even they are back to back, as shown in Figure 1.

\subsection{Model formulation}

Before building the mathematical model, we first clarify some assumptions:

"'» number of transhipment containers are considered to be available and deterministic within the planning horizon;

" " the berth position of the ship and the berthing time are deterministic;

"» operational level decisions, for example, the work order of yard cranes is not considered;

"»» the path travel time of container trucks follows normal distribution;

"') the waiting time at yard cranes and QCs is same for every container truck;

"» trucks in the yard are guided by an anticlockwise direction;

"» the congestion model is suitable for all the truck lanes.

\subsubsection{Notations}

Parameters:

$A$ - set of all the links in the yard network indexed by $a=(i, j), a=(i, j) \in A$;

subset of links in the loading path from subblock

$A_{s, v}^{L}-s$ to vessel $v, A_{s, v}^{L} \subseteq A$;

subset of links in the unloading path from vessel

$A_{v, s}^{U}-v$ to subblock $s, A_{v, s}^{U} \subseteq A$;

$N$ - set of all the nodes in the yard network; note that node $o \in N$ refers to origin and $d \in N$ refers to destination;

$N_{s}$ - subset of subblock nodes in the yard network, $N_{s} \subseteq N$;

$N_{v}$ - subset of berth nodes in the yard network, $N_{v} \subseteq N$

$P$ - set of the whole time periods indexed by $p$;

$P_{v}$ - subset of periods when vessel $v$ moors, $P_{v} \subseteq P$;

$S$ - set of all the subblocks indexed by $s$, note that $N_{s}$ refer to the corresponding node set of $S$ according to the yard network;

$S_{v}$ - subset of candidate subblocks that are assigned to vessel $v, S_{v} \subseteq S$;

$S_{i, j}$ - subset of subblocks that may have influence on the traffic in link $(i, j), S_{i, j} \subseteq S$;

$S_{g}$ - the group of subblocks, which belongs to block $g, S_{g} \subseteq S$;

$S_{\text {neigh }}$ - the pair of neighbour subblocks, e.g. $S_{\text {neigh }}=$ $\{21,39\}$ means subblock 21 and subblock 39 are neighbours, $S_{\text {neigh }} \in \mathbb{S}, \mathbb{S}$ is set of all the neighbour pairs;

$V$ - set of vessels indexed by $v$; note that $N_{v}$ refer to the corresponding node set of $V$ according to the yard network;

$V_{v}$ - subset of vessels that will load the containers that unloaded from vessel $v, V_{v} \subseteq V$;

$K$ - set of possible number of subblocks that are taking loading or unloading activities (indexed by $k$ ); $K=\{0,1, \ldots,|K|\}$ 
$\Omega$ - set of all the possible paths, and $\Omega_{u}^{o, d}$ means the $u$ path from $o$ to $d$; note that $u \subseteq A_{s, v}^{L}$ for loading path and $u \subseteq A_{v, s}^{U}$ for unloading path;

$m$ - number of vessels in the planning period;

$l_{v}$ - time length when vessel $v$ moors;

$n_{v}$ - number of subblocks that are assigned to vessel $v$;

$q_{\bar{v}, v}$ - number of containers, which are unloaded from vessel $\bar{v}$, stored in the yard, and then loaded on to vessel $v$ in future;

$t_{i, j, k}$ - the expected travel time when there are $k$ subblocks performing loading or unloading activities on link $(i, j)$;

$\varepsilon_{i, j, p}^{o, d}$ - equals to 1 if link $(i, j)$ is on the path $\Omega^{o, d}$ in period $p$;

$W_{L B}$ - lower bound of the minimum workload;

$W_{U B}$ - upper bound of the maximum workload;

$W_{Y C}$ - the maximum workload of a yard crane in a period;

$Y_{Y C}$ - the maximum number of yard cranes, which can work simultaneously in a block.

Decision variables:

$x_{u}^{o, d} \in\{0,1\}$ - set to one if $u$ path is selected from original $o$ to destination $d$;

$\beta_{s}^{v} \in\{0,1\}-$ set to one if subblock $s$ is assigned to vessel $v$; and zero otherwise;

$\eta_{s, p}^{L} \in\{0,1\}$ - set to one if subblock $s$ has loading activities in period $p$; zero otherwise;

$\eta_{s, p}^{U} \in\{0,1\}$ - set to one if subblock $s$ has unloading activities in period $p$; zero otherwise;

$\gamma_{i, j, k, p} \in\{0,1\}-$ set to one if there are $k$ subblocks that have loading or unloading activities along link $(i, j)$ in period $p$; and zero otherwise;

$\mu_{s, p} \in\{0,1\}$ - set to one if workload of subblock $v$ is high in period $p$; and zero otherwise;

$\lambda_{i, j, p} \geq 0$ - number of subblocks that have loading or unloading activities along link $(i, j)$ in period $p$;

$n_{o, d, p}^{L} \geq 0$ - number of loaded containers that go through path $\Omega^{o, d}$ in period $p$;

$n_{o, d, p}^{U} \geq 0$ - number of unloaded containers that go through path $\Omega^{o, d}$ in period $p$;

$\delta_{s, p}^{L} \geq 0$ - number of containers loaded from subblock $s$ in period $p$;

$\delta_{s, p}^{U} \geq 0$ - number of containers unloaded to subblock $s$ in period $p$.

\subsubsection{Model for optimizing container}

truck paths with uncertain travel time

$$
\min _{o \in N_{s}, d \in N_{v}, p \in P} \sum_{u \in \Omega^{o, d}}\left(t_{u}^{o, d}+z_{\alpha} \sigma_{u}^{o, d}\right) \cdot x_{u}^{o, d} \cdot n_{o, d, p}^{L}+
$$

$$
\sum_{o \in N_{v}, d \in N_{s}, p \in P} \sum_{u \in \Omega^{o, d}}\left(t_{u}^{o, d}+z_{\alpha} \sigma_{u}^{o, d}\right) \cdot x_{u}^{o, d} \cdot n_{o, d, p}^{U}
$$

subject to:

$$
\begin{aligned}
& \sum_{v \in V} \beta_{s}^{v} \leq 1, \forall s \in S ; \\
& \sum_{s \in S_{v}} \beta_{s}^{v}=n_{v}, \forall v \in V ; \\
& \sum_{s \in S \backslash S_{v}} \beta_{s}^{v}=0, \forall v \in V ; \\
& \sum_{u \subseteq A_{s, v}^{L}} x_{u}^{o, d}=1, \forall o \in N_{s}, d \in N_{v} ; \\
& \sum_{u \subseteq A_{v, s}^{U}} x_{u}^{o, d}=1, \forall o \in N_{v}, d \in N_{s} ; \\
& n_{i, j, p}^{L}=\sum_{v \in V, s \in S:(i, j) \in A_{s, v}^{L}, p \in P_{v}} \beta_{s}^{v} \cdot \frac{\sum_{\bar{v} \in V} q_{\bar{v}, v}}{n_{v} \cdot l_{v}}, \\
& \forall(i, j) \in A, \forall p \in P ;
\end{aligned}
$$$$
n_{i, j, p}^{U}=\sum_{\bar{v} \in V, s \in S:(i, j) \in A_{\bar{v}, s}^{U},} \sum_{p \in P_{\bar{v}}} \beta_{v \in V}^{v} \cdot \frac{q_{\bar{v}, v}}{n_{v} \cdot l_{v}},
$$$$
\forall(i, j) \in A, \forall p \in P \text {; }
$$$$
n_{o, d, p}^{L}=\sum_{i=o, j \in N} n_{i, j, p}^{L} \cdot \varepsilon_{i, j, p}^{o, d},
$$$$
\forall o \in N_{s}, \forall d \in N_{v}, \forall p \in P_{v} \text {; }
$$$$
n_{o, d, p}^{U}=\sum_{i \in N, j=d} n_{i, j, p}^{U} \cdot \varepsilon_{i, j, p}^{o, d},
$$$$
\forall o \in N_{v}, \forall d \in N_{s}, \forall p \in P_{v} \text {; }
$$$$
\eta_{s, p}^{L}=\sum_{v \in V: p \in P_{v}} \beta_{s}^{v}, \forall s \in S, \forall p \in P \text {; }
$$$$
\eta_{s, p}^{U} \geq \frac{\sum_{v \in V: p \in P_{v}} \sum_{\bar{v} \in V_{v}} \beta_{s}^{\bar{v}}}{m}, \forall s \in S, \forall p \in P ;
$$$$
\sum_{k \in K} \gamma_{i, j, k, p}=1, \forall(i, j) \in A, \forall p \in P \text {; }
$$$$
\sum_{k \in K} k \cdot \gamma_{i, j, k, p}=\lambda_{i, j, p}, \forall(i, j) \in A, \forall p \in P \text {; }
$$$$
\lambda_{i, j, p}=\sum_{s \in S_{i, j}}\left(\eta_{s, p}^{L}+\eta_{s, p}^{U}\right) \forall(i, j) \in A, \forall p \in P ;
$$$$
t_{u}^{o d}=\sum_{(i, j) \in u} \sum_{p \in P} \sum_{k \in K} t_{i, j, k} \cdot \gamma_{i, j, k, p},
$$

$\forall o \in N_{s}, \forall d \in N_{v}$ or $\forall o \in N_{v}, \forall d \in N_{s}$;

$\delta_{s, p}^{L}=\sum_{v \in V: p \in P_{v}} \beta_{s}^{v} \cdot \frac{\sum_{\bar{v} \in V} q_{\bar{v}, v}}{n_{v} \cdot l_{v}}, \forall s \in S, \forall p \in P$; 


$$
\begin{aligned}
& \delta_{s, p}^{U}=\sum_{\bar{v} \in V: p \in P_{\bar{v}}} \sum_{v \in V} \beta_{s}^{v} \cdot \frac{q_{\bar{v}, v}}{n_{v} \cdot l_{v}}, \forall s \in S, \forall p \in P \\
& \mu_{s, p} \cdot W_{L B} \leq \delta_{s, p}^{L}+\delta_{s, p}^{U} \leq W_{L B}+\mu_{s, p} \cdot\left(W_{U B}-W_{L B}\right) \\
& \forall s \in S, \forall p \in P ; \\
& \sum_{s \in S_{n e i g h}} \mu_{s, p} \leq 1, \forall S_{n e i g h} \in \mathbb{S}, \forall p \in P ; \\
& \sum_{s \in S_{g}}\left(\delta_{s, p}^{L}+\delta_{s, p}^{U}\right) \leq W_{Y C} \cdot Y_{Y C}, \forall S_{g} \subseteq S, \forall p \in P ; \\
& x_{u}^{o, d} \in\{0,1\}, \\
& \forall o \in N_{s}, \forall d \in N_{v} \text { or } \forall o \in N_{v}, \forall d \in N_{s} ; \\
& \beta_{s}^{v} \in\{0,1\}, \forall v \in V, \forall s \in S ; \\
& \eta_{s, p}^{L}, \eta_{s, p}^{U}, \mu_{s, p} \in\{0,1\}, \forall s \in S, p \in P ; \\
& \delta_{s, p}^{L}, \delta_{s, p}^{U} \geq 0, \forall s \in S, \forall p \in P ; \\
& \lambda_{i, j, p}, n_{i, j, p}^{L}, n_{i, j, p}^{U} \geq 0 \quad \forall(i, j) \in A, \forall p \in P \\
& n_{o, d, p}^{L}, n_{o, d, p}^{U} \geq 0, \forall o \in N_{s}, \forall d \in N_{v}, p \in P \text { or } \\
& \forall o \in N_{v}, \forall d \in N_{s}, p \in P ; \\
& \gamma_{i, j, k, p} \in\{0,1\}, \forall(i, j) \in A, \forall p \in P, \forall s \in S \\
& \forall \\
& \forall
\end{aligned}
$$

Objective (14) is to minimize the total travel time of containers going through the paths, which include the loading and unloading paths between vessels and subblocks. Constraints (15) express that each subblock is allocated to no more than one vessel. Constraints (16) and (17) ensure the number of subblocks allocated to vessel $v$. Constraints (18) and (19) ensure that only one path is selected for each loading/unloading process. Constraints (20) and (21) indicate the number of loaded and unloaded containers passing through link $(i, j)$ in period $\mathrm{c}$. Constraints (22) and (23) define the number of loaded and unloaded containers through path $\Omega^{o, d}$ in period $p$. Constraints (24) and (25) define the binary variable to ensure that whether a subblock has loading or unloading activities in a period. In the right side of Constraint (25), $\sum_{v \in V: p \in P_{v}} \sum_{\bar{v} \in V_{v}} \beta_{s}^{\bar{v}}$ means the number of all the vessels that use subblock $s$ to unload containers. As $\eta_{s, p}^{U}$ is binary variable, $\sum_{v \in V: p \in P_{v}} \sum_{\bar{v} \in V_{v}} \beta_{s}^{\bar{v}}$ should be divided by $m$. If $\eta_{s, p}^{U}$ is positive, $\eta_{s, p}^{U}$ equals to one; otherwise, it is zero. Constraints (26) and (27) combine the binary variables $\gamma_{i, j, k, p}$ with the integer variables $\lambda_{i, j, p}$, which denotes the number of working subblocks on link $(i, j)$ in period $(i, j)$. Constraint (28) define the relationship between $\lambda_{i, j, p}$ and $\eta_{s, p}^{L}, \eta_{s, p}^{U}$. Constraint (29) express that the expectation of path travel time is the sum of link travel time considering yard congestion. Constraints (30) and (31) respectively calculate the number of containers, which are loaded from and unloaded to a subblock during a time period. Constraint (32) ensure the workload activities in each subblock is either high (i.e., $\mu_{s, p}=1$ ) or low (i.e., $\mu_{s, p}=0$ ). Constraint (33) guarantee that high-workload activity should not happen between two neighbour subblocks simultaneously. Constraints (32) and (33) are derived from a common workload balancing protocol to mitigating congestion (see subsection 2.1.5). Constraint (34) restrict that the workload activities within a block should not exceed the number of yard cranes. Usually, there are two yard cranes in each block. Constraints (35)-(41) define decision variables.

\subsubsection{Linearization for the model}

Objective (14) is nonlinear. To linearize the objective so that it could be solved by commercial solvers, some auxiliary decision variables and constraints are added. The new variables $\omega_{o, d, u, p}^{L}$ and $\omega_{o, d, u, p}^{U}$ are defined to take the place of $x_{u}^{o, d} n_{o, d, p}^{L}$ and $x_{u}^{o, d} n_{o, d, p}^{U}$, respectively:

"» $\omega_{o, d, u, p}^{L} \geq 0$ the number of loaded containers from origin $o$ to destination $d$ in period $p$, if $u$ path is selected, $\omega_{o, d, u, p}^{L}=0$ otherwise;

"» $\omega_{o, d, u, p}^{U} \geq 0$ the number of unloaded containers from origin $o$ to destination $d$ in period $p$, if $u$ path is selected, $\omega_{o, d, u, p}^{L}=0$ otherwise.

The model could be changed to:

$$
\begin{gathered}
\min _{o \in N_{s},}^{\mathrm{M}_{\text {OCTP-UTT: }}:} \sum_{o \in N_{v},}\left(t_{u}^{o, d}+z_{\alpha} \sigma_{u}^{o, d}\right) \cdot \omega_{o, d, u, p}^{L}+ \\
\sum_{s \in P}\left(t_{u}^{o, d}+z_{\alpha} \sigma_{u}^{o, d}\right) \cdot \omega_{o, d, u, p}^{U}
\end{gathered}
$$

subject to Constraints (16)-(42).

$$
\begin{aligned}
& \omega_{o, d, u, p}^{L} \geq n_{o, d, p}^{L}+\left(x_{u}^{o, d}-1\right) \cdot M, \\
& \forall o \in N_{s}, \forall d \in N_{v}, \forall u \in \Omega^{o, d}, \forall p \in P ; \\
& \omega_{o, d, u, p}^{U} \geq n_{o, d, p}^{U}+\left(x_{u}^{o, d}-1\right) \cdot M, \\
& \forall o \in N_{v}, \forall d \in N_{s}, \forall u \in \Omega^{o, d}, \forall p \in P ; \\
& \omega_{o, d, u, p}^{L}, \omega_{o, d, u, p}^{U} \geq 0, \\
& \forall o \in N_{s}, \forall d \in N_{v}, \forall u \in \Omega^{o, d}, p \in P \text { or } \\
& \forall o \in N_{v}, \quad \forall d \in N_{s}, \forall u \in \Omega^{o, d}, \forall p \in P .
\end{aligned}
$$

In Constraints (43) and (44), $M$ is a large number to guarantee the linearization that:

$$
\omega_{o, d, u, p}^{L}= \begin{cases}n_{o, d, p}^{L}, & \text { if } x_{u}^{o, d}=1 \\ 0, & \text { if } x_{u}^{o, d}=0,\end{cases}
$$

and

$$
\omega_{o, d, u, p}^{U}=\left\{\begin{array}{ll}
n_{o, d, p}^{U}, & \text { if } x_{u}^{o, d}=1 \\
0, & \text { if } x_{u}^{o, d}=0 .
\end{array} .\right.
$$




\section{Model solution}

Considering the proposed $\mathrm{M}_{\text {OCTP-UTT }}$ model is complex, it cannot be solved by analytical algorithm methods such as column generation, branch and price algorithm efficiently, because it is difficult to define the objective value of columns. Other widely used analytical algorithm method such as the dynamic programming is also not valid to solve the model because the decision process is hard to be divided into stages. Therefore, in this paper, the SCE-UA and $\mathrm{A}^{*}$ algorithm are introduced to solve the proposed $\mathrm{M}_{\text {OCTP-UTT }}$ model. The SCE-UA algorithm is an effective evolution algorithm, which is similar to genetic algorithm. This method was firstly applied to optimize the parameters of the hydrologic models (Duan et al. 1994). Because of the advantage of global optimization, it was applied to other areas. Yu et al. (2020) used SCE-UA algorithm on the Subordinate Net Points Layout Optimization of Express Enterprise. The results were feasible and had excellent robustness. While $A^{*}$ algorithm is an efficient algorithm in finding shortest path between any two given nodes, it is also a heuristic algorithm firstly applied in Hart et al. (1968). In our problem, we consider the uncertain travel time talked in subsection 2.1 and propose a reliable shortest path algorithm based on $\mathrm{A}^{*}$, called RSPP-A*

\subsection{SCE-UA algorithm to work out the yard template planning problem}

The SCE-UA algorithm is a global optimization algorithm that integrates the advantages of deterministic search, random search, and competition evolution. It performs well in global search performance and efficiency of multi-parameter combination. In this section, we use SCE-UA algorithm to work out the yard template planning problem, in which the algorithm decides the assignment of containers between vessels and subblocks. The pseudo-code of SCE-UA algorithm is presented in Algorithm 1. Step 1 to step 10 is initial process, which defines the parameters (complex $p$ and points in $p$ ) and find a feasible solution. Step 11 to step 17 is heuristic searching process. In the process, the genetic and mutation step is done in a CCE algorithm (see Algorithm 2) to generate new solutions. The algorithm stops until the solution satisfies a check convergence procedure.

The CCE algorithm is the crucial part in SCE-UA. The main purpose of CCE is to generate better solutions than that in last iteration. The pseudo-code of CCE algorithm is presented in Algorithm 2. Step 1 is initial process to set the parameter (the iteration $\sigma$ and evolution $\tau$ ). Step 3 to step 5 define the probability of being selected from parent solution. Step 7 to step 11 generate offspring solution from the selected parent solution. Step 12 to 27 check the dominance rules to see if the offspring solution could dominance the parent solution.

\section{2. $A^{*}$ algorithm to find reliable shortest path}

This section presents a multicriteria $\mathrm{A}^{*}$ algorithm, named RSPP-A ${ }^{*}$, to find the reliable shortest path in the proposed yard nsetwork. Given the origin $o$ and destination $d$ of the containers obtained from subsection 3.1 and the on-time probability $\alpha$, the RSPP- $\mathrm{A}^{*}$ algorithm could find a reli-

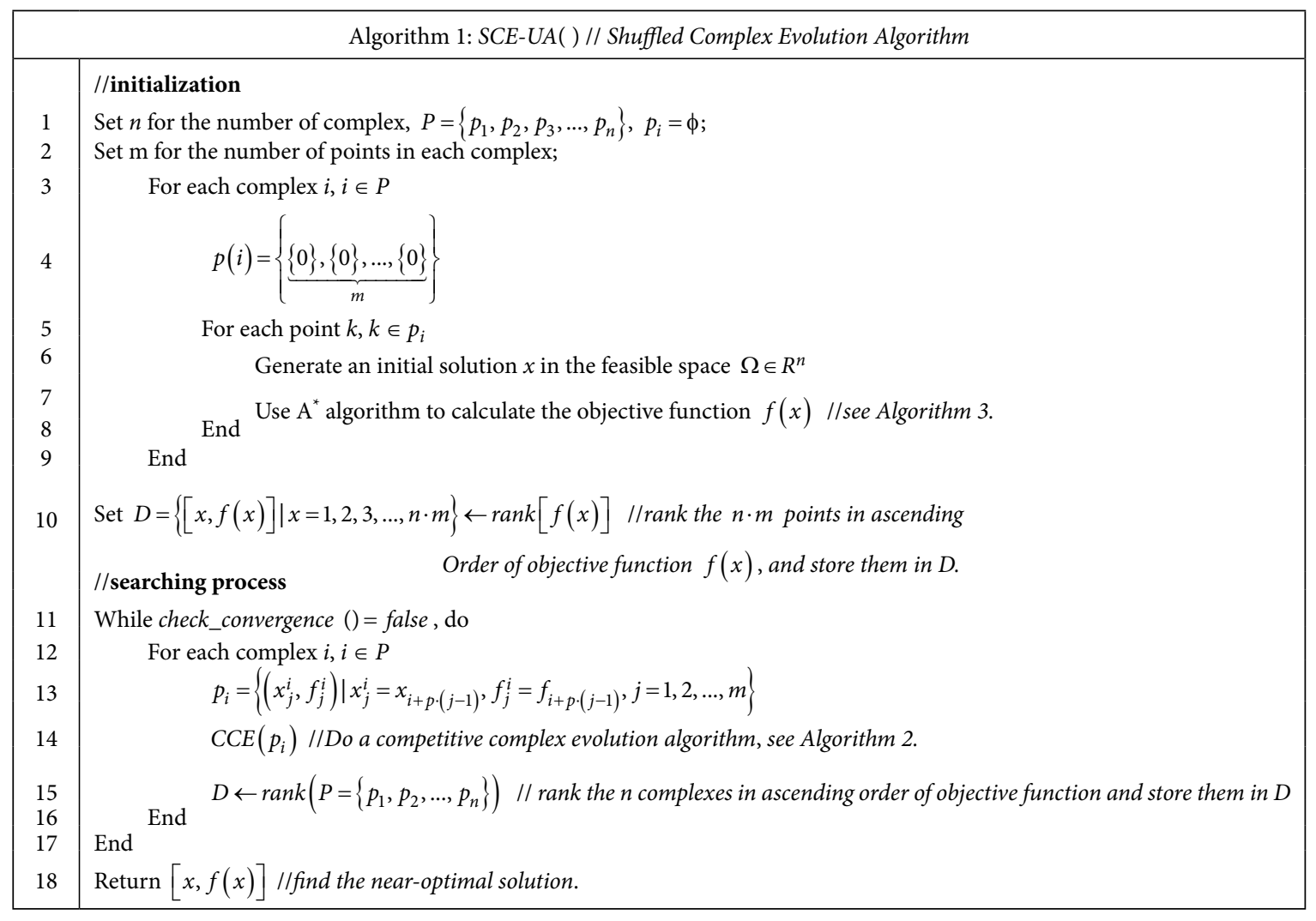




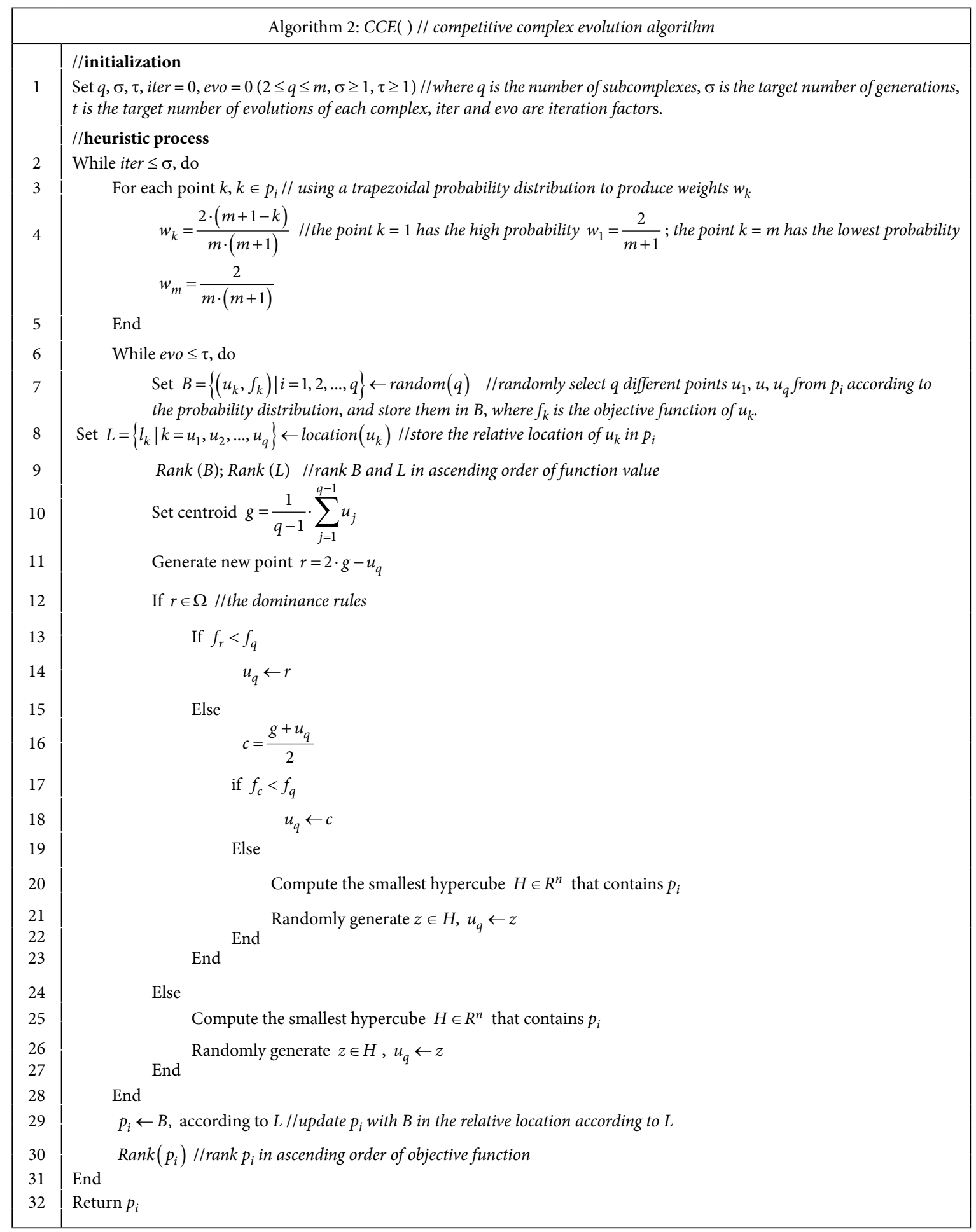

able shortest path for container trucks. The pseudo-code of RSPP-A ${ }^{*}$ algorithm is given in Algorithm 3. Step 1 to step 10 initialize the parameters by setting an open list for unexamined nodes and a close list for examined nodes. The idea of RSPP-A ${ }^{*}$ is consistently transforming nodes from open list to close list until finding the destination $d$. Step 11 to step 14 calculate the estimated distance function $F(i)=x(i)+y(i)$. Here $x(i)$ is the reliable distance from origin $o$ to node $i$ considering the yard congestion and correlation between adjacent links, while $y(i)$ is an estimated distance from node $i$ to destination $d$. We use Manhattan distance to calculate $y(i)$. Manhattan distance only considers the horizontal and vertical moves to estimate a distance from node $i$ to destination $d . y(i)$ may not be a real distance but could give a direction for path extension. Step 15 to step 16 decide the node that could be transformed to the close list. Step 17 to step 28 check the dominance. Node with smaller distance could dominate the same node with larger distance. For example, $F^{1}(i) \leq F^{2}(i), F^{1}(i)$ dominates $F^{2}(i)$. 


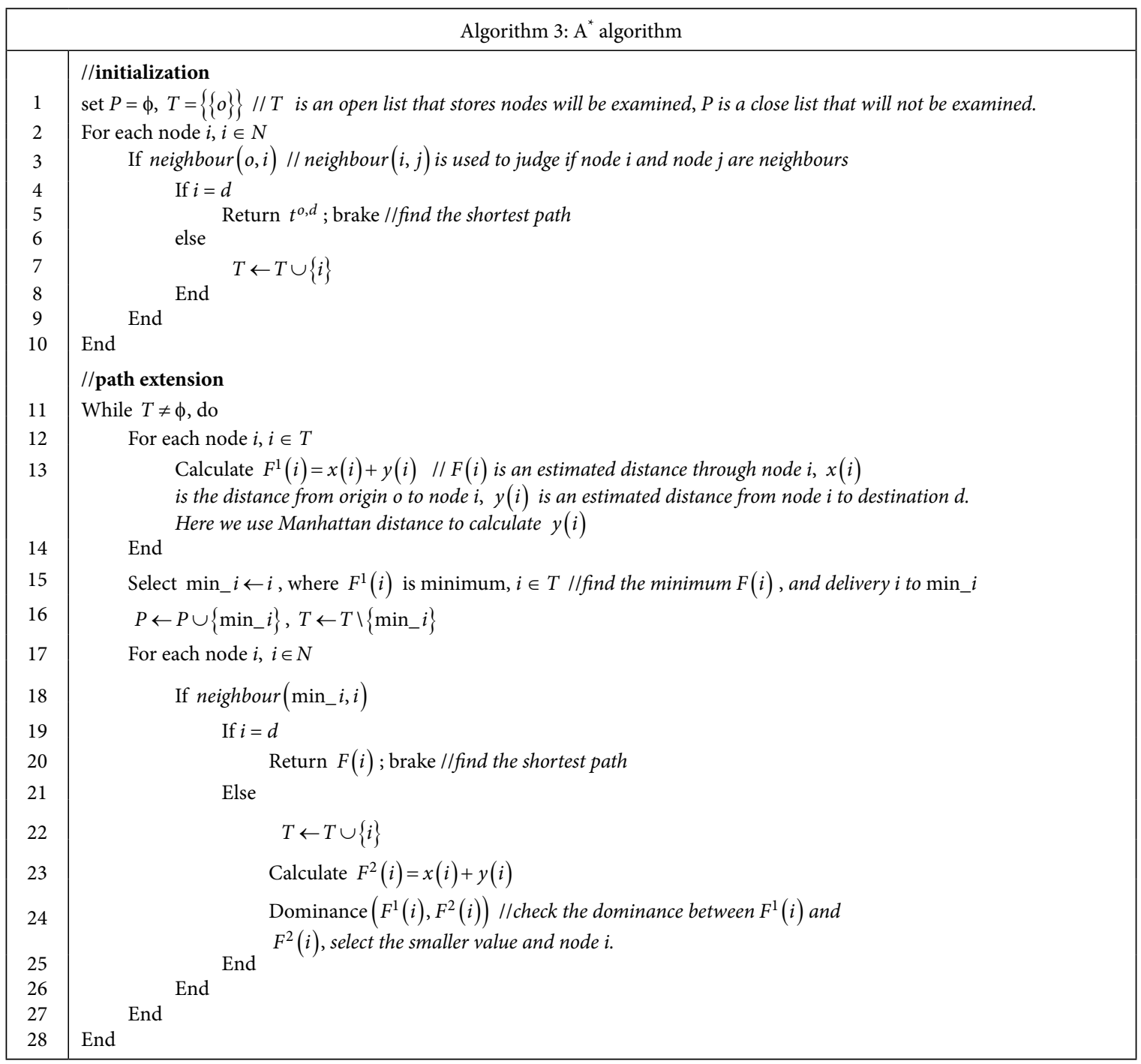

\section{Case study}

In case study, one yard of Dalian container port is used to demonstrate the applicability of the proposed algorithm. In addition, several experiments are performed to validate the effectiveness of the proposed model.

\subsection{The specification of case study}

The planning horizon is 1 week considering the cycle of container liner transportation typically measures in weeks. The planning horizon is divided into 168 time periods and each time period is set to be $1 \mathrm{~h}$. It is assumed that each vessel has specific arrival time made in advance, so the subset periods for each vessel with loading/unloading activities at port could be determined. In this case, the yard is considered to have 144 subblocks at most, and the layout of one yard in Dalian container port is shown in Figure 5.

Each block is 6 containers (TEU) deep and 36 containers long. There are at most two yard cranes in one block, which is coincident with the real situations in ports. The block is further divided into six subblocks. Each subblock is six containers long and five containers high. The capacity of each subblock is $180(=6 \cdot 6 \cdot 5)$ TEUs. The width of horizontal and vertical passing lanes in the yard are set to be 30 and $70 \mathrm{~m}$ respectively. Each vessel is set to have 4 or 5 subblocks to store its containers. The transhipped containers in each vessel can be loaded to at most 5 other vessels. Then according to the data of Dalian container port, the number of containers to be unloaded and loaded by a yard crane in one hour is 24 TEUs. When calculating the travel time for each link by Equation (5), the parameters are set as follow: the average speed of trucks is $8 \mathrm{~m} / \mathrm{s}$; the acceleration or deceleration rate of trucks is $2 \mathrm{~m} / \mathrm{s}^{2}$. The turning speed at the cross is $4 \mathrm{~m} / \mathrm{s}$. The handing time for a container is $150 \mathrm{~s}$. The parameters are estimated by Dalian container port. For the yard cranes' capacity in the port, the maximum value during one period is set to 30 . For mitigating the yard congestions, the condition of high workload is set to a range of $[15,30)$, while the low workload is $[0,15)$. 


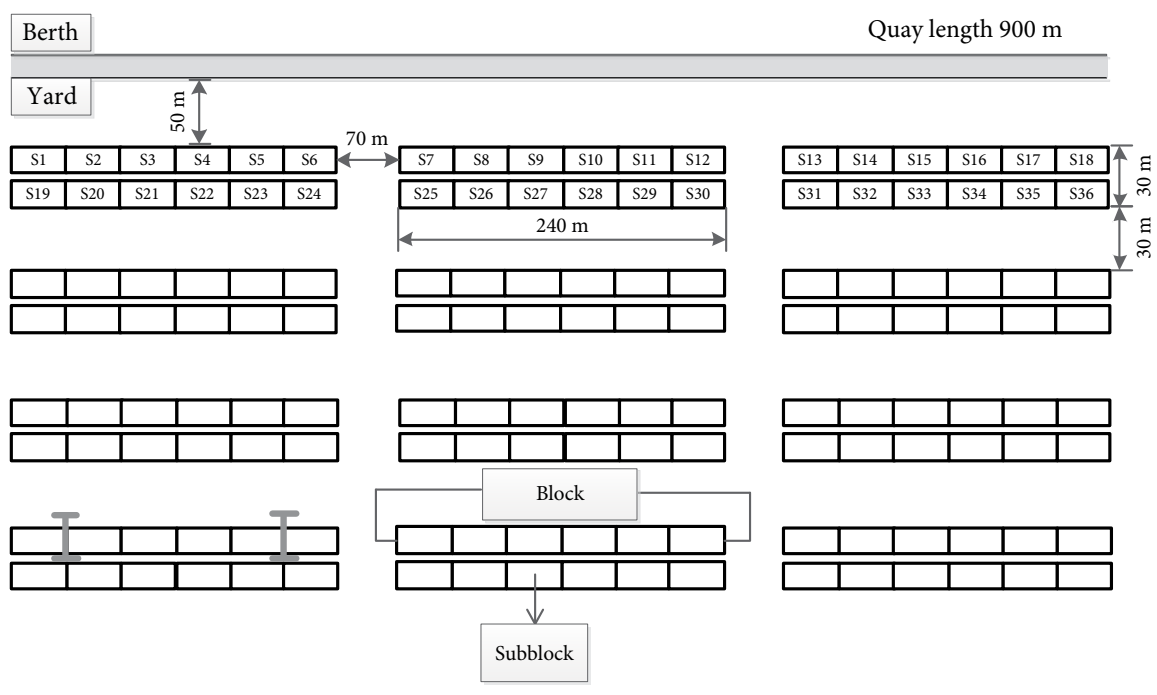

Figure 5. The layout of one yard in Dalian container port

\subsection{Evaluating the efficiency of the proposed solution method}

\subsubsection{Comparing with the optimal results of small-scale instances}

In order to evaluate the performance of the SCE-UA algorithm, we compare the results of the SCE-UA algorithm with the CPLEX. Table 2 shows the comparison results between two methods on objective value and computation time. As we can see, the gap is small. Moreover, the solution time of the SCE-UA algorithm is much shorter than the CPLEX. The results in Table 2 validate the efficiency of the SCE-UA algorithm on small-scale instances.

\subsubsection{Comparing with the situation of not considering the correlation among adjacent links}

The previous studies mainly minimize the total length or time of traffic routes. In other words, it is assumed that the expected travel time of each $(i, j)$ link is determined before the optimization, and is not influenced by the number of container trucks on each link (Zhen et al. 2011; Jiang et al. 2012). One contribution in this study is the consideration of the correlation among adjacent links when finding the shortest path of container trucks. We would like to explore the influence of considering and not considering the correlation among adjacent links in Table 3. According to Equation (8), $\sigma$ is evaluated by the data we researched in Dalian port. We investigated the travel time of 15 sequential container trucks at each link and then got the triangular matrix, which contains the variance and covariance of adjacent links. Table 3 shows the results of the two situations, in which column 2 is the results of $\mathrm{M}_{\text {OCTP-UTT }}$ model considering the link correlation, and column 3 is the results of $\mathrm{M}_{\text {OCTP-UTT }}$ model not considering link correlation.

In Table 3, the average gap value varies from 3 to $9 \%$. The results show that a yard with the size of 144 subblocks can save the total truck travel time by $9 \%$ when its yard template is optimized by considering the correlation among adjacent links. Furthermore, some interesting results could be observed. Figure 6 presents that the gap increases with the growth of subblocks. While in Figure 7, the gap decreases with the increased number of vessels, because the feasible solution decreases and the shortest paths are relatively fixed. As a result, the gap increases with the number of subblocks and the number of vessels increased simultaneously, which can be seen in Figure 8. The three figures indicate that the influence of increased subblocks to the shortest path plays a more important role than that of increased vessels.

\subsubsection{Testing the performance of the proposed algorithm by benchmark}

In this section, the cases about 20 subblocks and 4 vessels are also solved by the algorithm used in Zhen (2016), which is taken as a benchmark. In Zhen (2016), the authors applied a SWO for changing the sequence of vessels so as to improve the quality of solutions. As the yard template problem is to assign subblocks to vessels, the sequence of the vessel is important for the assignment. In their algorithm, they firstly calculated the path travel time relate to each vessel, and then swap two consecutive vessels if the path travel time of the former is lower than the latter. After swap operation, they will get new solutions. The algorithm stops after observing a number of unchanged solutions. The results of the proposed algorithm and the SWO based algorithm are compared in Table 4. The performance differences between these two heuristics are small. It is observed that the proposed SCEUA algorithm has longer CPU time (20.35 s on average, compared to16.30 s) but near optimal solution (305530 on average, compared to 305579).

\subsubsection{Sensitivity analysis for the closeness degree of $k$-neighbouring links}

The correlation among adjacent links is considered by building a $k$-neighbouring network for link $(i, j)$, denoted as $G_{i j}^{k}=\left(N_{i j}^{k}, A_{i j}^{k}, \Psi_{i j}^{k}\right)$, satisfying $X_{i j}^{q w} \leq k,(q, w) \in A_{i j}^{k}$. $X_{i j}^{q w}$ is the topological distance between link $(i, j)$ and link $(q, w)$. The sensitivity analysis of the closeness degree is shown in Table 5. 
Table 2. Comparison of the optimal solution for the proposed solution method

\begin{tabular}{|c|c|c|c|c|c|}
\hline \multirow{2}{*}{ Case } & \multicolumn{2}{|c|}{ Best results by CPLEX } & \multicolumn{2}{|c|}{ SCE-UA algorithm } & \multirow{2}{*}{ Gap $[\%$} \\
\hline & $\mathrm{BR}_{\text {CPLEX }}$ & CPU time $[\mathrm{s}]$ & $\mathrm{BR}_{\text {SCE-UA }}$ & CPU time $[\mathrm{s}]$ & \\
\hline 1 & 306551 & 1061 & 306551 & 15 & 0.00 \\
\hline 2 & 310415 & 1525 & 310415 & 25 & 0.00 \\
\hline 3 & 314279 & 1558 & 314279 & 23 & 0.00 \\
\hline 4 & 316211 & 1479 & 316211 & 15 & 0.00 \\
\hline 5 & 302687 & 2199 & 302687 & 20 & 0.00 \\
\hline 6 & 304560 & 1413 & 304681 & 15 & 0.04 \\
\hline 7 & 307371 & 1638 & 307647 & 21 & 0.09 \\
\hline 8 & 308638 & 2903 & 308730 & 19 & 0.03 \\
\hline 9 & 301502 & 3773 & 301562 & 27 & 0.02 \\
\hline 10 & 305231 & 3915 & 305322 & 21 & 0.03 \\
\hline 11 & 300609 & 3355 & 300639 & 22 & 0.01 \\
\hline 12 & 304444 & 2001 & 304778 & 15 & 0.11 \\
\hline 13 & 310181 & 1736 & 310646 & 19 & 0.15 \\
\hline 14 & 312992 & 1631 & 313649 & 17 & 0.21 \\
\hline 15 & 309531 & 3059 & 309654 & 18 & 0.04 \\
\hline 16 & 306568 & 5255 & 306843 & 20 & 0.09 \\
\hline 17 & 307753 & 5573 & 307968 & 18 & 0.07 \\
\hline 18 & 296315 & 2845 & 296492 & 23 & 0.06 \\
\hline 19 & 291341 & 2304 & 291457 & 24 & 0.04 \\
\hline 20 & 290142 & 2786 & 290401 & 16 & 0.09 \\
\hline
\end{tabular}

Notes:

"» the case consists of 24 subblocks and 4 vessels;

"» $\mathrm{Gap}=\frac{\mathrm{BR}_{\mathrm{SCE}-\mathrm{UA}}-\mathrm{BR}_{\mathrm{CPLEX}}}{\mathrm{BR}_{\mathrm{CPLEX}}} \cdot 100 \%$.

Table 3. Comparison of situations with and without considering the correlation among links

\begin{tabular}{|c|c|c|c|}
\hline \multirow{2}{*}{$\begin{array}{c}\text { Case } \\
\text { scale }\end{array}$} & $\begin{array}{c}\mathrm{M}_{\text {OCTP-UTT model }} \\
\text { considering link } \\
\text { correlation }\end{array}$ & $\begin{array}{c}\mathrm{M}_{\text {OCTP-UTT model }} \\
\text { not considering } \\
\text { link correlation }\end{array}$ & \multirow{2}{*}{ Gap [\%] } \\
\cline { 2 - 3 } & AVG BR SCE-UA $_{\text {N }}$ & AVG BR $_{\text {NON-CORR }}$ & \\
\hline $24-4$ & 305714 & 316842 & 3.64 \\
\hline $24-6$ & 354242 & 366475 & 3.45 \\
\hline $24-8$ & 409534 & 422578 & 3.19 \\
\hline $36-6$ & 465278 & 483146 & 3.84 \\
\hline $36-8$ & 501403 & 519941 & 3.70 \\
\hline $36-9$ & 541576 & 560624 & 3.52 \\
\hline $36-12$ & 642887 & 664124 & 3.30 \\
\hline $48-8$ & 631477 & 660145 & 4.54 \\
\hline $48-12$ & 726632 & 757140 & 4.20 \\
\hline $48-16$ & 835864 & 867654 & 3.80 \\
\hline $72-12$ & 957921 & 1014547 & 5.91 \\
\hline $72-18$ & 1117636 & 1175655 & 5.19 \\
\hline $72-24$ & 1342180 & 1406533 & 4.79 \\
\hline $96-16$ & 1304893 & 1401697 & 7.42 \\
\hline $96-24$ & 1493546 & 1593462 & 6.69 \\
\hline $96-32$ & 1762462 & 1864421 & 5.79 \\
\hline $144-24$ & 1943267 & 2127440 & 9.48 \\
\hline $144-36$ & 2304521 & 2510462 & 8.94 \\
\hline
\end{tabular}

Note: The case scale $24-4$ means the cases consists of 24 subblocks and 4 vessels, etc.
Table 4. Performance of $\mathrm{BR}_{\mathrm{SCE}-\mathrm{UA}}$ in benchmark instances

\begin{tabular}{|c|c|c|c|c|}
\hline \multirow{2}{*}{ Case } & \multicolumn{2}{|c|}{ SWO based algorithm } & \multicolumn{2}{|c|}{ SCE-UA algorithm } \\
\hline & $\mathrm{BR}_{\text {SWO }}$ & CPU time $[\mathrm{s}]$ & $\mathrm{BR}_{\mathrm{SCE}-\mathrm{UA}}$ & CPU time [s] \\
\hline 1 & 306551 & 13 & 306551 & 17 \\
\hline 2 & 310415 & 23 & 310415 & 25 \\
\hline 3 & 314279 & 12 & 314279 & 16 \\
\hline 4 & 316211 & 13 & 316211 & 23 \\
\hline 5 & 302687 & 11 & 302687 & 20 \\
\hline 6 & 304660 & 18 & 304681 & 17 \\
\hline 7 & 307371 & 19 & 307647 & 23 \\
\hline 8 & 308638 & 19 & 308730 & 19 \\
\hline 9 & 301554 & 18 & 301562 & 27 \\
\hline 10 & 305279 & 22 & 305322 & 21 \\
\hline 11 & 300652 & 18 & 300639 & 22 \\
\hline 12 & 304563 & 11 & 304778 & 15 \\
\hline 13 & 310796 & 12 & 310646 & 26 \\
\hline 14 & 312774 & 14 & 313649 & 19 \\
\hline 15 & 309531 & 16 & 309654 & 18 \\
\hline 16 & 306959 & 19 & 306843 & 19 \\
\hline 17 & 308412 & 23 & 307968 & 21 \\
\hline 18 & 297043 & 15 & 296492 & 23 \\
\hline 19 & 291834 & 14 & 291457 & 17 \\
\hline 20 & 291387 & 16 & 290401 & 19 \\
\hline AVG & 305579 & 16.30 & 305530 & 20.35 \\
\hline
\end{tabular}

Note: the case consists of 20 subblocks and 4 vessels. 


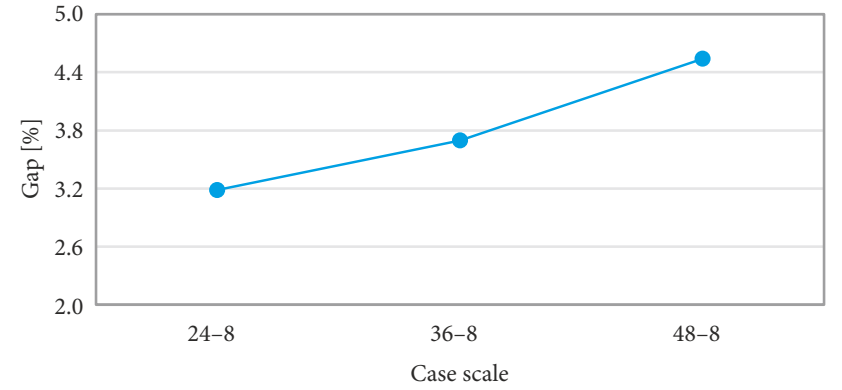

Figure 6. The trend of gap with the increasing subblocks

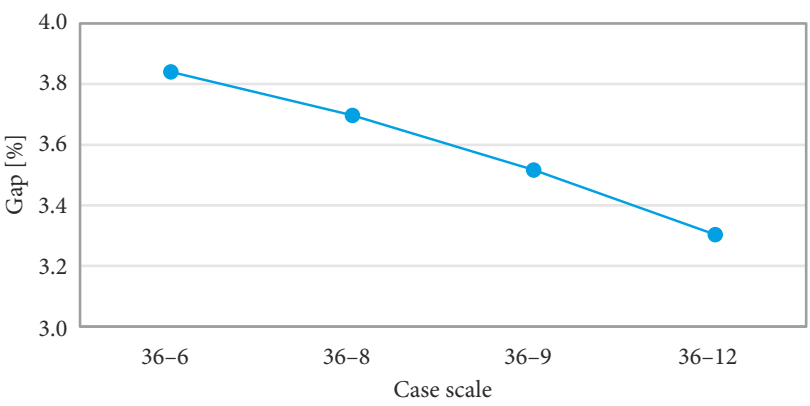

Figure 7. The trend of gap with the increasing vessels

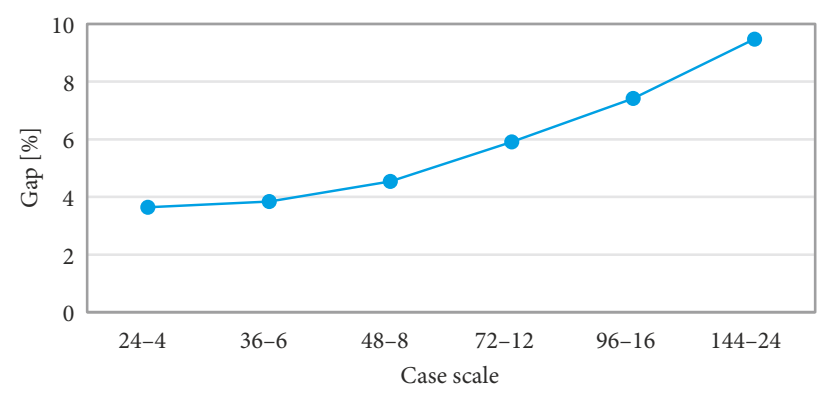

Figure 8 . The trend of gap with the increasing subblocks and vessels

As can be seen in Table 5, with the increase of the closeness degree, the average results decreases. In case 24-4 and case 48-8, the results decrease sharply when $k=$ $1,2,3$, but when $k=4,5$, the results hardly decrease. $k=3$ is the break point. Similar phenomena can be seen in case $72-12$ and case 144-24, but the break point becomes $k=4$. The reason is, in small scale cases, the correlation among adjacent links is not quite obvious, and the covariance nearly equals 0 when the closeness $k=4$ or $k=5$. Therefore, the results in small scale cases decrease a little when $k=4,5$. While in large scale cases such as $72-12$ and 144-24, the path becomes complicated and easy to be influenced by adjacent links, so the results decrease sharply when $k=1,2,3,4$, and hardly decrease when $k=5$. For simplicity, we choose $k=3$ in this paper.

\subsubsection{Sensitivity analysis for yard scales and number of yard trucks}

In this section, cases with different yard scales and number of yard trucks are designed. Each case is done by 5 simulation runs. If the yard scale changes, the travel distance of container trucks changes accordingly. The results are shown in Table 6. The gap of results decreases with
Table 5. Results of cases with different closeness degree

\begin{tabular}{|c|c|c|}
\hline Case scale & $\begin{array}{c}\text { Closeness } \\
\text { degree } k\end{array}$ & AVG BR \\
\hline \multirow{4}{*}{$24-4$ SCE-UA }
\end{tabular}

Note: the case scale $24-4$ means the cases consists of 24 subblocks and 4 vessels, etc.

Table 6. Results of cases with different yard scales

\begin{tabular}{|c|c|c|c|c|c|}
\hline $\begin{array}{l}\text { Case } \\
\text { scale }\end{array}$ & $\begin{array}{c}\text { Number of subblocks } \\
\text { in one block }\end{array}$ & $\begin{array}{l}\text { Mean } \\
\text { value }\end{array}$ & $\begin{array}{l}\text { Min } \\
\text { value }\end{array}$ & $\begin{array}{l}\text { Max } \\
\text { value }\end{array}$ & $\begin{array}{l}\text { Gap } \\
{[\%]}\end{array}$ \\
\hline \multirow{7}{*}{$4-4$} & 4 & 249453 & 243824 & 256577 & 5.23 \\
\hline & 5 & 279546 & 274356 & 286630 & 4.47 \\
\hline & 6 & 305714 & 301338 & 311843 & 3.49 \\
\hline & 8 & 368943 & 361356 & 371854 & 2.91 \\
\hline & 10 & 435585 & 431320 & 439379 & 1.87 \\
\hline & 12 & 463246 & 459111 & 465407 & 1.37 \\
\hline & 15 & 527520 & 525894 & 530619 & 0.90 \\
\hline \multirow{7}{*}{$8-4$} & 4 & 312493 & 308203 & 324221 & 5.20 \\
\hline & 5 & 368905 & 361894 & 376965 & 4.16 \\
\hline & 6 & 429534 & 420439 & 434218 & 3.28 \\
\hline & 8 & 499543 & 495655 & 508230 & 2.54 \\
\hline & 10 & 553940 & 550283 & 556932 & 1.21 \\
\hline & 12 & 593063 & 590893 & 596220 & 0.90 \\
\hline & 15 & 624952 & 622956 & 627018 & 0.65 \\
\hline \multirow{7}{*}{$8-8$} & 4 & 522371 & 517303 & 529209 & 2.30 \\
\hline & 5 & 584910 & 579281 & 590366 & 1.91 \\
\hline & 6 & 631477 & 625683 & 638524 & 2.05 \\
\hline & 8 & 714859 & 710235 & 718090 & 1.11 \\
\hline & 10 & 750922 & 747128 & 754006 & 0.92 \\
\hline & 12 & 793274 & 790283 & 796212 & 0.75 \\
\hline & 15 & 852038 & 850173 & 854293 & 0.48 \\
\hline
\end{tabular}

Notes:

"» the case scale $4-4$ means the cases consists of 4 blocks and 4 vessels, etc.;

"» Gap $=\frac{\max \text { value }- \text { min value }}{\text { min value }} \cdot 100 \%$. 
the increase of subblocks in one block. The reason is that less truck interruptions happen on the path if the length of block increases, because the containers are dispersed in the subblocks and avoid congestion. Therefore, the travel time of container trucks would become reliable. Furthermore, the number of blocks seems to have no apparent effects to the gap when comparing the case 4 blocks and 4 vessels to the case 8 blocks and 4 vessels. The number of vessels could reduce the gap apparently when comparing the case 8 blocks and 4 vessels to the case 8 blocks and 8 vessels. The port operators could consider to enlarge the number of subblocks in one block.

Next, cases with different number of container trucks entering into yard are designed. The results are shown in Table 7. It seems that the optimal number of container trucks is different in different case scale. The optimal num-

Table 7. Results of cases with different number of container trucks

\begin{tabular}{|c|c|c|}
\hline Case scale & $\begin{array}{c}\text { Number of } \\
\text { container trucks }\end{array}$ & AVG BR SCE-UA $_{\text {A }}$ \\
\hline \multirow{9}{*}{$24-4$} & 50 & 341680 \\
\hline & 75 & 319034 \\
\hline & 100 & 308482 \\
\hline & 125 & 304952 \\
\hline & 150 & 305714 \\
\hline & 175 & 310368 \\
\hline & 200 & 329487 \\
\hline & 225 & 351082 \\
\hline & 250 & 398495 \\
\hline \multirow{9}{*}{$48-8$} & 50 & 771464 \\
\hline & 75 & 720633 \\
\hline & 100 & 689051 \\
\hline & 125 & 652482 \\
\hline & 150 & 631477 \\
\hline & 175 & 646392 \\
\hline & 200 & 661543 \\
\hline & 225 & 680372 \\
\hline & 250 & 703189 \\
\hline \multirow{9}{*}{$72-12$} & 50 & 1540330 \\
\hline & 75 & 1284954 \\
\hline & 100 & 1103943 \\
\hline & 125 & 1028492 \\
\hline & 150 & 957921 \\
\hline & 175 & 913782 \\
\hline & 200 & 874859 \\
\hline & 225 & 842924 \\
\hline & 250 & 850922 \\
\hline
\end{tabular}

Note: the case scale $24-4$ means the cases consists of 24 subblocks and 4 vessels, etc. ber of container truck is $125 \mathrm{vel} / \mathrm{h}, 150 \mathrm{vel} / \mathrm{h}$ and $225 \mathrm{vel} / \mathrm{h}$ for the case scale of 24 subblocks 4 vessels, 48 subblocks and 8 vessels and 72 subblocks and 12 vessels, respectively.

\subsubsection{Sensitivity analysis for confidence level $a$}

The other contribution of this study is considering the probability of arriving at the destination within the expected travel time. As the yard operation is complex, e.g. the yard crane moves from one side to the other side at uncertain time, container trucks may arrive at the destination early or late. The confidence level $\alpha \in(0,1)$ is the probability that container trucks arrive at the destination within the expected travel time. The on-time arrival probability $\alpha$ represents port operator's attitude towards risks of being late ( $\alpha>0.5, \alpha=0.5$ and $\alpha<0.5$ for risk-averse, risk-neutral, and risk-seeking attitudes, respectively). The value of $\alpha$ can be predetermined based on port operators' purpose. $\alpha=0.5$ means the operators take no account of potential risk in the yard. In addition, $\alpha>0.5$ means the operators pay attention to the potential risk, which often happens in heavy workload ports. $\alpha<0.5$ means the operators ignore the potential risk, which often happens in low workload ports. As shown in Table 8 , the $\mathrm{BR}_{\mathrm{SCE}-\mathrm{UA}}$ is different with different confidence level $\alpha$. When $\alpha=0.1$, the case 11 obtains the minimum travel time cost. But the case 20 obtains the minimum travel time cost when $\alpha=0.5$.

Table 8. Results of cases with different confidence level $\alpha$

\begin{tabular}{|c|c|c|c|}
\hline \multirow{2}{*}{ Case } & \multicolumn{3}{|c|}{ BR $_{\text {SCE-UA }}$} \\
\cline { 2 - 4 } & $\alpha=0.1$ & $\alpha=0.5$ & $\alpha=0.9$ \\
\hline 1 & 295576 & 306551 & 317526 \\
\hline 2 & 305526 & 310415 & 315304 \\
\hline 3 & 311883 & 314279 & 316675 \\
\hline 4 & 311178 & 316211 & 321244 \\
\hline 5 & 290139 & 302687 & 315235 \\
\hline 6 & 303358 & 304681 & 305762 \\
\hline 7 & 304285 & 307647 & 310457 \\
\hline 8 & 304875 & 308730 & 312401 \\
\hline 9 & 300534 & 301562 & 302470 \\
\hline 10 & 298049 & 305322 & 312413 \\
\hline 11 & $\mathbf{2 8 4 5 6 1}$ & 300639 & 316657 \\
\hline 12 & 304206 & 304778 & 304682 \\
\hline 13 & 309107 & 310646 & 311255 \\
\hline 14 & 311970 & 313649 & 314014 \\
\hline 15 & 305014 & 309654 & 314048 \\
\hline 16 & 303381 & 306843 & 309755 \\
\hline 17 & 296939 & 307968 & 318567 \\
\hline 18 & 295083 & 296492 & 297547 \\
\hline 19 & 292395 & 291457 & 295463 \\
\hline 20 & 294081 & $\mathbf{2 9 0 4 0 1}$ & 294721 \\
\hline
\end{tabular}

Note: the case consists of 24 subblocks and 4 vessels. 


\section{Conclusions}

The paper studies the optimization of the container truck paths with uncertain travel time in container ports. In the proposed model, the link travel time influenced by the yard congestion is formulated as a basic work. In addition, the reliable shortest path related with the correlation among adjacent links is discussed in our problem. A small illustrative example shows that the shortest path may change considering the correlation among adjacent links. It is necessary to emphasize that the container truck path optimization should not be separately treated. The problem is influenced by the yard template planning.

Considering the intricate workload in a yard, this paper proposes the confidence level $\alpha$ to cover different situations in container ports. In the real application, it could provide different strategies in different conditions (peak season or low season), which is helpful for container port operation and schedule. A mixed-integer programming model is proposed to minimize the total travel time of container trucks in the yard. The combination of SCEUA and $\mathrm{A}^{*}$ algorithm is developed to solve the model. The cases are presented to validate the availability of the model. The results show that $9 \%$ of the travel time of container trucks can be saved when the correlation among adjacent links is considered. It may raise an inspired idea to yard management and equipment scheduling in ports, especially in transhipment ports.

However, there are limitations on the current model. For example, the container truck drivers may have different driving behaviour. It may be better to use car following model to calculate the expected travel time on each link. Besides, the arrival time of each vessel is determined before the yard template planning. In practice, the arrival time and operation time of vessels are stochastic. These limitations will be further studied in our future researches.

\section{Funding}

This research was supported by National Natural Science Foundation of China (Grants No U1811463, 71961137008), the State Key Laboratory of Structural Analysis for Industrial Equipment (Grant No S18307), and partially funded by Beijing Advanced Innovation Center for Big Data and Brain Computing of Beihang Universtity.

\section{Author contributions}

Jiaming Liu and Bin Yu conceived the study and were responsible for the design and development of the data analysis.

Jiaming Liu, Baozhen Yao were responsible for data collection and analysis.

Wenxuan Shan and Baozhen Yao were responsible for data interpretation.

Yao Sun wrote the first draft of the article.

\section{Disclosure statement}

Authors are required to include a statement at the end of their article to declare whether or not they have any competing financial, professional, or personal interests from other parties.

\section{References}

Cao, J. X.; Lee, D.-H.; Chen, J. H.; Shi, Q. 2010. The integrated yard truck and yard crane scheduling problem: Benders' decomposition-based methods, Transportation Research Part E: Logistics and Transportation Review 46(3): 344-353. https://doi.org/10.1016/j.tre.2009.08.012

Chan, K. S.; Lam, W. H. K.; Tam, M. L. 2009. Real-time estimation of arterial travel times with spatial travel time covariance relationships, Transportation Research Record: Journal of the Transportation Research Board 2121: 102-109. https://doi.org/10.3141/2121-11

Chang, D.; Jiang, Z.; Yan, W.; He, J. 2010. Integrating berth allocation and quay crane assignments, Transportation Research Part E: Logistics and Transportation Review 46(6): 975-990. https://doi.org/10.1016/j.tre.2010.05.008

Chang, T.-S.; Nozick, L. K.; Turnquist, M. A. 2005. Multiobjective path finding in stochastic dynamic networks, with application to routing hazardous materials shipments, Transportation Science 39(3): 383-399. https://doi.org/10.1287/trsc.1040.0094

Chen, A.; Ji, Z. 2005. Path finding under uncertainty, Journal of Advanced Transportation 39(1): 19-37. https://doi.org/10.1002/atr.5670390104

Chen, B. Y.; Lam, W. H. K.; Sumalee, A.; Li, Z.-L. 2012. Reliable shortest path finding in stochastic networks with spatial correlated link travel times, International Journal of Geographical Information Science 26(2): 365-386.

https://doi.org/10.1080/13658816.2011.598133

Chen, B. Y.; Lam, W. H. K.; Sumalee, A.; Li, Q.; Tam, M. L. 2014. Reliable shortest path problems in stochastic time-dependent networks, Journal of Intelligent Transportation Systems: Technology, Planning, and Operations 18(2): 177-189. https://doi.org/10.1080/15472450.2013.806851

Chen, C.; Tian, Z.; Yao, B. 2019. Optimization of two-stage location-routing-inventory problem with time-windows in food distribution network, Annals of Operations Research 273(1-2): 111-134. https://doi.org/10.1007/s10479-017-2514-3

Chen, L.; Langevin, A.; Lu, Z. 2013. Integrated scheduling of crane handling and truck transportation in a maritime container terminal, European Journal of Operational Research 225(1): 142-152. https://doi.org/10.1016/j.ejor.2012.09.019

Chen, X.; Zhou, X.; List, G. F. 2011. Using time-varying tolls to optimize truck arrivals at ports, Transportation Research Part E: Logistics and Transportation Review 47(6): 965-982. https://doi.org/10.1016/j.tre.2011.04.001

Duan, Q.; Sorooshian, S.; Gupta, V. K. 1994. Optimal use of the SCE-UA global optimization method for calibrating watershed models, Journal of Hydrology 158(3-4): 265-284. https://doi.org/10.1016/0022-1694(94)90057-4

Fan, L.; Wilson, W. W.; Dahl, B. 2012. Congestion, port expansion and spatial competition for US container imports, Transportation Research Part E: Logistics and Transportation Review 48(6): 1121-1136. https://doi.org/10.1016/j.tre.2012.04.006 
Han, Y.; Lee, L. H.; Chew, E. P.; Tan, K. C. 2008. A yard storage strategy for minimizing traffic congestion in a marine container transshipment hub, OR Spectrum 30(4): 697-720. https://doi.org/10.1007/s00291-008-0127-6

Hart, P. E.; Nilsson, N. J.; Raphael, B. 1968. A formal basis for the heuristic determination of minimum cost paths, IEEE Transactions on Systems Science and Cybernetics 4(2): 100-107. https://doi.org/10.1109/TSSC.1968.300136

He, J.; Huang, Y.; Yan, W.; Wang, S. 2015. Integrated internal truck, yard crane and quay crane scheduling in a container terminal considering energy consumption, Expert Systems with Applications 42(5): 2464-2487.

https://doi.org/10.1016/j.eswa.2014.11.016

Huang, B.; Wu, Q.; Zhan, F. B. 2007. A shortest path algorithm with novel heuristics for dynamic transportation networks, International Journal of Geographical Information Science 21(6): 625-644. https://doi.org/10.1080/13658810601079759

Jiang, X.; Lee, L. H.; Chew, E. P.; Han, Y.; Tan, K. C. 2012. A container yard storage strategy for improving land utilization and operation efficiency in a transshipment hub port, European Journal of Operational Research 221(1): 64-73. https://doi.org/10.1016/j.ejor.2012.03.011

Jin, J. G.; Lee, D.-H.; Hu, H. 2015. Tactical berth and yard template design at container transshipment terminals: A column generation based approach, Transportation Research Part E: Logistics and Transportation Review 73: 168-184.

https://doi.org/10.1016/j.tre.2014.11.009

Kaveshgar, N.; Huynh, N. 2015. Integrated quay crane and yard truck scheduling for unloading inbound containers, International Journal of Production Economics 159: 168-177. https://doi.org/10.1016/j.ijpe.2014.09.028

Kim, K. H.; Bae, J. W. 1998. Re-marshaling export containers in port container terminals, Computers \& Industrial Engineering 35(3-4): 655-658.

https://doi.org/10.1016/S0360-8352(98)00182-X

Kim, K. H.; Kim, K. Y. 2007. Optimal price schedules for storage of inbound containers, Transportation Research Part B: Methodological 41(8): 892-905.

https://doi.org/10.1016/j.trb.2007.02.001

Lee, D.-H.; Jin, J. G. 2013. Feeder vessel management at container transshipment terminals, Transportation Research Part E: Logistics and Transportation Review 49(1): 201-216. https://doi.org/10.1016/j.tre.2012.08.006

Lee, H. L.; Chew, E. P.; Tan, K. C.; Han, Y. 2006. An optimization model for storage yard management in transshipment hubs, OR Spectrum 28(4): 539-561.

https://doi.org/10.1007/s00291-006-0045-4

Lu, Y.; Le, M. 2014. The integrated optimization of container terminal scheduling with uncertain factors, Computers \& Industrial Engineering 75: 209-216.

https://doi.org/10.1016/j.cie.2014.06.018

Maloni, M.; Paul, J. A. 2013. Evaluating capacity utilization options for US west coast container ports, Transportation Journal 52(1): 52-79.

https://doi.org/10.5325/transportationj.52.1.0052

Meng, Q.; Wang, S.; Andersson, H.; Thun, K. 2014. Containership routing and scheduling in liner shipping: overview and future research directions, Transportation Science 48(2): 265280. https://doi.org/10.1287/trsc.2013.0461

Moccia, L.; Cordeau, J.-F.; Monaco, M. F.; Sammarra, M. 2009. A column generation heuristic for a dynamic generalized assignment problem, Computers \& Operations Research 36(9): 2670-2681. https://doi.org/10.1016/j.cor.2008.11.022
Moorthy, R.; Teo, C.-P. 2006. Berth management in container terminal: the template design problem, OR Spectrum 28(4): 495-518. https://doi.org/10.1007/s00291-006-0036-5

Nie, Y.; Wu, X. 2009. Shortest path problem considering on-time arrival probability, Transportation Research Part B: Methodological 43(6): 597-613. https://doi.org/10.1016/j.trb.2009.01.008

Nikolova, E. 2009. Strategic Algorithms. PhD Thesis. Massachusetts Institute of Technology, MA, US. 212 p. Available from Internet: https://dspace.mit.edu/handle/1721.1/54673

Nishimura, E.; Imai, A.; Janssens, G. K.; Papadimitriou, S. 2009. Container storage and transshipment marine terminals, Transportation Research Part E: Logistics and Transportation Review 45(5): 771-786.

https://doi.org/10.1016/j.tre.2009.03.003

Nishimura, E.; Imai, A.; Papadimitriou, S. 2005. Yard trailer routing at a maritime container terminal, Transportation Research Part E: Logistics and Transportation Review 41(1): 53-76. https://doi.org/10.1016/j.tre.2003.12.002

Peng, Z.; Shan, W.; Jia, P.; Yu, B.; Jiang, Y.; Yao, B. 2020. Stable ride-sharing matching for the commuters with payment design, Transportation 47(1): 1-21.

https://doi.org/10.1007/s11116-018-9960-x

Peng, Z.; Shan, W.; Guan, F.; Yu, B. 2016. Stable vessel-cargo matching in dry bulk shipping market with price game mechanism, Transportation Research Part E: Logistics and Transportation Review 95: 76-94.

https://doi.org/10.1016/j.tre.2016.08.007

Roy, D.; Gupta, A.; De Koster, R. B. M. 2016. A non-linear traffic flow-based queuing model to estimate container terminal throughput with AGVs, International Journal of Production Research 54(2): 472-493. https://doi.org/10.1080/00207543.2015.1056321

Shan, W.; Yan, Q.; Chen, C.; Zhang, M.; Yao, B.; Fu, X. 2019. Optimization of competitive facility location for chain stores, Annals of Operations Research 273(1-2): 187-205. https://doi.org/10.1007/s10479-017-2579-z

Shao, H.; Lam, W. H. K.; Chan, K. S. 2004. The problem of searching the reliable path for transportation networks with uncertainty, in Proceeding of 9th Conference of the Hong Kong Society for Transportation Studies, Hong Kong, China, 226-234.

Vis, I. F. A.; De Koster, R. 2003. Transshipment of containers at a container terminal: an overview, European Journal of Operational Research 147(1): 1-16.

https://doi.org/10.1016/S0377-2217(02)00293-X

Wang, S.; Liu, Z.; Bell, M. G. H. 2015. Profit-based maritime container assignment models for liner shipping networks, Transportation Research Part B: Methodological 72: 59-76. https://doi.org/10.1016/j.trb.2014.11.006

Yao, B.; Chen, C.; Song, X.; Yang, X. 2019a. Fresh seafood delivery routing problem using an improved ant colony optimization, Annals of Operations Research 273(1): 163-186. https://doi.org/10.1007/s10479-017-2531-2

Yao, B.; Chen, C.; Zhang, L.; Yu, B.; Wang, Y. 2019b. Allocation method for transit lines considering the user equilibrium for operators, Transportation Research Part C: Emerging Technologies 105: 666-682. https://doi.org/10.1016/j.trc.2018.09.019

Yu, B.; Wang, H.; Shan, W.; Yao, B. 2018. Prediction of bus travel time using random forests based on near neighbors, Computer-Aided Civil and Infrastructure Engineering 33(4): 333-350. https://doi.org/10.1111/mice.12315 
Yu, B.; Wang, H.; Song, X.; Zhao, Z.; Tian, Z.; Yao, B. 2020. Optimising subordinate net points layout of express enterprise with SCE-UA algorithm, Proceedings of the Institution of Civil Engineers - Transport 173(1): 51-58.

https://doi.org/10.1680/jtran.16.00092

Zeng, W.; Church, R. L. 2009. Finding shortest paths on real road networks: the case for $\mathrm{A}^{\star}$, International Journal of Geographical Information Science 23(4): 531-543. https://doi.org/10.1080/13658810801949850

Zhang, C.; Liu, J.; Wan, Y.-W.; Murty, K. G.; Linn, R. J. 2003. Storage space allocation in container terminals, Transportation Research Part B: Methodological 37(10): 883-903. https://doi.org/10.1016/S0191-2615(02)00089-9

Zhang, M.; Batta, R.; Nagi, R. 2009. Modeling of workflow congestion and optimization of flow routing in a manufacturing/warehouse facility, Management Science 55(2): 267-280. https://doi.org/10.1287/mnsc.1080.0916

Zhen, L. 2016. Modeling of yard congestion and optimization of yard template in container ports, Transportation Research Part B: Methodological 90: 83-104. https://doi.org/10.1016/j.trb.2016.04.011

Zhen, L. 2015. Tactical berth allocation under uncertainty, European Journal of Operational Research 247(3): 928-944. https://doi.org/10.1016/j.ejor.2015.05.079

Zhen, L. 2013. Yard template planning in transshipment hubs under uncertain berthing time and position, Journal of the Operational Research Society 64(9): 1418-1428. https://doi.org/10.1057/jors.2012.108

Zhen, L.; Chew, E. P.; Lee, L. H. 2011. An integrated model for berth template and yard template planning in transshipment hubs, Transportation Science 45(4): 483-504. https://doi.org/10.1287/trsc.1100.0364

Zhen, L.; Yu, S.; Wang, S.; Sun, Z. 2019. Scheduling quay cranes and yard trucks for unloading operations in container ports, Annals of Operations Research 273(1-2): 455-478.

https://doi.org/10.1007/s10479-016-2335-9 